\title{
Die Erhaltung der bäuerlichen Kulturlandschaft der Alpen agrar- und gesellschaftspolitische Instrumente und Hindernisse
}

\section{Working Paper}

Author(s):

Rieder, Peter

Publication date:

1991

Permanent link:

https://doi.org/10.3929/ethz-a-000662506

Rights / license:

In Copyright - Non-Commercial Use Permitted

Originally published in:

Schriftenreihe / ETH Zürich, Institut für Agrarwirtschaft 1991 


\author{
ETH Zürich
}

Institut für Agrarwirtschaft/Institut d'économie rurale

\title{
Schriftenreihe Publications
}

$1991 / 2$

DIE ERHALTUNG DER BÄUERLICHEN KULTURLANDSCHAFT DER ALPEN - AGRAR- UND GESELLSCHAFTSPOLITISCHE INSTRUMENTE UND HINDERNISSE

Peter Rieder

Oktober 1991

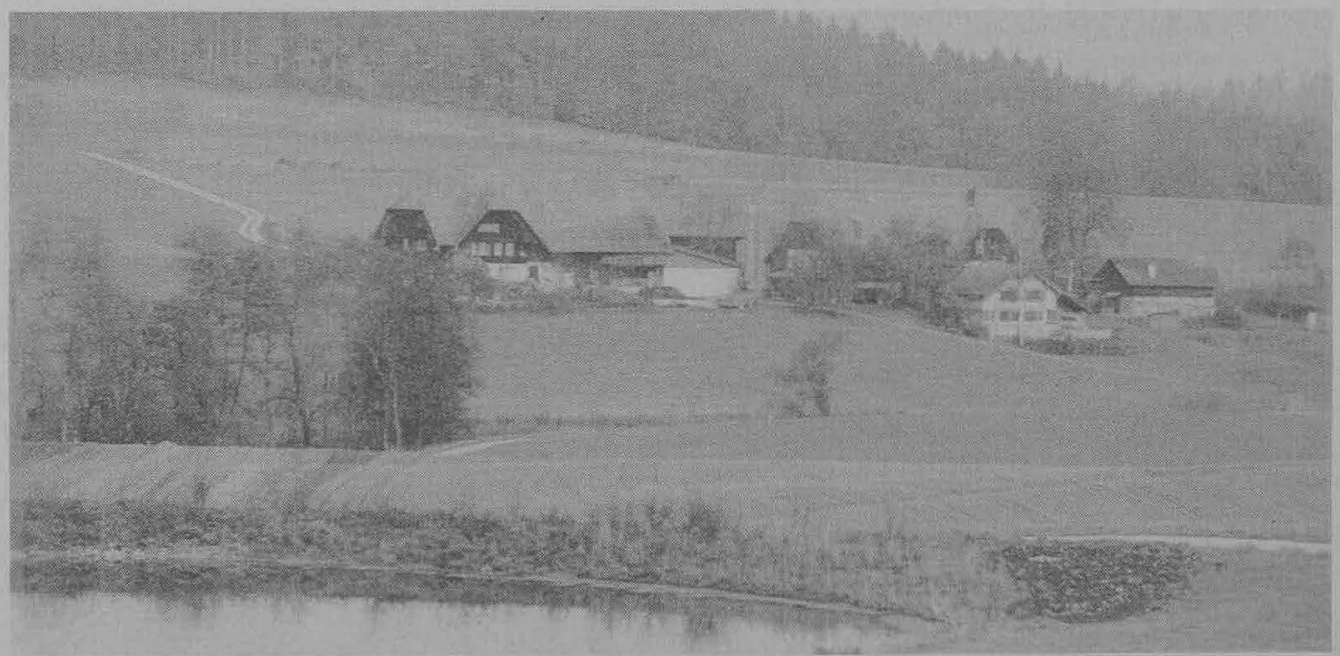

ETH-Zentrum, 8092 Zürich 


\section{$1991 / 2$}

DIE ERHALTUNG DER BÄUERLICHEN KULTURLANDSCHAFT DER ALPEN - AGRAR - UND GESELLSCHAFTSPOLITISCHE INSTRUMENTE UND HINDERNISSE

$$
\text { Peter Rieder }
$$

Oktober 1991

Adresse des Verfassers:

Institut für Agrarwirtschaft ETH-Zentrum

8092 Zürich 


\section{Die Erhaltung der bäuerlichen Kulturlandschaft der Alpen - agrar- und gesellschaftspolitische Instrumente und Hindernisse}

Peter Rieder

\section{EINLEITUNG}

Vieles ist in unserer Zeit in Bewegung geraten: Die Sowjetunion und die osteuropäischen Kleinstaaten stehen in einem unerwarteten wirtschaftlichen und politischen Umbruch. In Westeuropa laufen bedeutende, wenn auch schwierige Integrationsverhandlungen (EWR, EG-Erweiterung). Im Rahmen der Uruguay-Runde des GATT sollen unter anderem die total verzerrten internationalen Agrarmärkte neu geordnet werden. Ein weiterer Verhandlungsgegenstand der GATT- und vieler anderer Konferenzen ist die Verbesserung der Verhältnisse in den Entwicklungsländern und deren Marktzutritts in Europa und den USA. In diesem weltpolitischen Umfeld sind wir bestrebt, in diesem Beitrag Wege aufzuzeigen, wie im Alpenraum Kultur und Kulturlandschaft erhalten, aber auch gezielt gefördert und gestaltet werden könnten. Im Gegensatz etwa zu Problemlösungen in Entwicklungsländern und in Osteuropa befinden wir uns in einem äusserst günstigen Umfeld von wohlhabenden Ländern und reichen Gesellschaften.

Den unmittelbaren Anstoss zu diesem Beitrag gaben die oben erwähnten Agrarverhandlungen im Rahmen des GATT, des EWR und der EG-Integration.

Angesichts der um sich greifenden Befürchtung, der Alpenraum bzw. die Berglandwirtschaft werde das erste opfer der internationalen Verhandlungsergebnisse sein, geraten Politiker und Funktionäre scheinbar in Bedrängnis. Gelegentlich finden sie angebliche Lösungen in alten Begriffen wie "Europa der Regionen" oder in der Schöpfung neuer Begriffe mit altem Inhalt wie "Multifunktionalität" der Landwirtschaft.

Ausgehend vom erwähnten weltweiten Umfeld und basierend auf historischen und agrarökonomischen Kenntnissen skizziere ich im folgenden einen Weg, wie die anerkannten Zielsetzungen für unseren Alpenraum möglichst effizient und nachhaltig zu erreichen sind.

Im Ablauf werde ich zuerst einige historische Hinweise anbringen, um dann die heutige Situation zu charakterisieren. Es folgt weiter ein Kommentar zur heutigen Agrarpolitik für das Berggebiet. Im zukunftsbezogenen Teil gehe ich zuerst auf die individuelle Zielsetzung des Einzelbauern ein, dann auf die öffentlichen Ziele bzw. Funktionen. Dann wird nach deren Uebereinstimmung gefragt und gezeigt, was die Agrarund Regionalpolitik dazu beiträgt. Anschliessend werden die Fächer zu den GATT- und EG-Verhandlungsergebnissen geöffnet. Im Abschnitt 7 behandeln wir eingehend die Multifunktionali- 
tät und leiten davon ab, was in Zukunft passieren könnte bzw. was zu tun wäre, um diese effizient und nachhaltig aufrecht zu erhalten.

\section{LANDSCHAFT UND LANDWIRTSCHAFT IM ALPENRAUM IN HISTORISCHER SICHT}

\section{a) Besiedlung im Mittelalter}

In Geschichtsbüchern können wir nachlesen, dass im Mittelalter, also im 12. und 13. Jahrhundert, in Europa ein starkes Bevölkerungswachstum stattfand. Das fruchtbare Land in den Tieflagen wurde knapp, waren doch viele Hänge noch bewaldet und die Flusslandschaften nichts als Sümpfe voll mit gefährlichen Krankheitserregern (Bundi, 1982). Klimatologen wissen auch zu berichten, dass zwischen etwa 750 und 1200 das Klima in den Alpentälern gegenüber heute milder und trockener gewesen sei (Löhr, 1971). Diese Zeit wird als das "kleine mittelalterliche Optimum" bezeichnet.

$\mathrm{Zu}$ Beginn dieser Epoche waren es noch die Romanen, die Nachkommen aus der rätischen Kolonie, die die Voralpen und die Ostalpen besiedelten. In der $\mathrm{Zwischenzeit} \mathrm{gerieten} \mathrm{sie} \mathrm{unter}$ die Herrschaft der Alemannen, so insbesondere durch Karl den Grossen um 800, die die tieferen Lagen der Alpentäler besiedelt hatten. Grafen, Vögte, Freiherren, Fürstbischöfe und Fürstäbte übten mit kaiserlicher Erlaubnis die Macht über die ihnen vom Kaiser überlassenen oder gekauften Talschaften aus. Die Bauern besassen zwar bereits relativ grosse Freiheiten, waren aber kriegs-, zins- und abgabepflichtig. Um die Situation treffend zu charakterisieren, ist der Hinweis wichtig, dass in jener Zeit die Bevölkerungsdichte im Alpenraum sehr gering war, und dass nur die tieferen und sonnigen Lagen der Alpentäler besiedelt waren. Aus Beschreibungen der Königshöfe weiss man, dass auf diesen Gütern in besten Lagen vor allem Ackerbau und sogar Weinbau betrieben wurde (Bundi, 1982).

Mit dem Bevölkerungswachstum gingen auch im Alpenraum Völkerwanderungen einher. Für den schweizerischen Alpenraum, aber auch das Vorarlberg, waren die Walserwanderungen von grosser Bedeutung. Mehrere Gründe sollen diesen historisch letzten Schub einer Völkerwanderung ausgelöst haben: Einmal die starke Vergrösserung der Bevölkerung im Oberwallis, für die der karge Boden damals kaum genug hergab. Dann sollen die Leute im Oberwallis unter der strengen Herrschaft der Fürstbischöfe von Sitten gelitten haben. Gleichzeitig gewann in den Ostalpen, vor allem im Kanton Graubünden, der Alpentransitverkehr an Bedeutung. Um die Alpenübergänge zu sichern, um zu roden und um in der Folge die Hochtäler zu besiedeln, lockten die damaligen Edlen von Vaz, vor allem Walther IV von Vaz (1284), und dessen Sohn Donat von Vaz ( 1337) die Walser an, damit jene Hochtäler besiedelt würden. Es sind dies überall jene höchstgelegenen Täler gewesen, die heutzutage wegen des Wintertourismus bekannt sind (Davos, Arosa, Klosters, Hinterrhein, Valsertal, Kleines Walsertal u.a.m.) (Zinsli, 1968). 
Grosse Ländereien waren in jener zeit noch im Besitz von Klöstern. Man muss aber auch diesem Zustand seine positive Seite abringen. Sie trugen die Ausbildungsstätten der Elite, und gleichzeitig waren sie die Verbreiter des Fortschritts in der Landwirtschaft und in der Viehhaltung. Belegt sind vor allem die Vieh- und Pferdezucht im Kloster Einsiedeln, aber auch die Klöster Engelberg, St.Gallen und Disentis sowie viele andere im Tirol und im Grossen Walsertal waren als Kulturverbreiter im wahrsten Sinne des Wortes tätig. Das "ora et labora" der Benediktinermönche war es also, das am Beginn dessen stand, was wir heute als Kulturlandschaft im Alpenraum bewundern.

\section{b) Bauernunruhen und Physiokraten}

Wie bisher dargelegt wurde, genossen im Gegensatz zu weiten Teilen des übrigen Europas die Bauern der Alpengebiete schon im Mittelalter relativ grosse Freiheiten. Die Macht der Städte und des Landadels bestand vor allem darin, dass (zu) hohe Zinsen und Abgaben eingezogen wurden. So hat der Reformator Zwingli nicht nur gegen das Reislaufen, sondern auch gegen $\mathrm{zu}$ hohe Zinsen gepredigt. Unter seinem Einfluss wurde 1529 ein Maximal-Zinsfuss von 5\% als "Fruchtteil" festgelegt. Dieser Zinssatz ist später allerdings in ein Zinsminimum umgewandelt worden (Howald, 1946).

Die eigentlichen Bauernunruhen, wie sie als Folge der durch Martin Luther ausgelösten sozialen Bewegung in deutschen Gebieten stattfanden, sind im Alpenraum nur in Einzelfällen aufgetreten. In Oesterreich kam es in Bergbaugebieten zu Bauernaufständen. Oft war der Auslöser auch hier die Neue Lehre, weil die Bauern und Bergbauarbeiter sich daraus Hilfe aus ihrer wirtschaftlichen Bedrängnis erhofften. In der Schweiz sind die Bauernaufstände im Entlebuch und Emmenthal eine Folge des Dreissigjährigen Krieges gewesen. Infolge inflationärer Tendenzen erhöhten die Städte Luzern und Bern die Zins- und Abgabenforderungen. Dadurch verarmten die Bauern so stark, dass sie sich unter der Führung von Nikolaus Leuenberger gegen die Städte auflehnten. Die Unruhen wurden niedergeschlagen, und der Anführer wurde hingerichtet.

Vom Merkantilismus, wie er vor allem vom Absolutismus in Frankreich betrieben wurde und dies zu "zerfetzten und zerlumpten" Bauern führte (Abel, 1951), blieben die Alpenregionen weitgehend verschont, weil sie nur schwach in die Geldwirtschaft der Städte eingebunden waren. Der Vorteil der Naturalwirtschaft lag in der Stetigkeit der geforderten Waren- und Dienstleistungen nach Menge und Wert über weite Zeiträume hinweg. So wusste man, was ein Hof nachhaltig zu leisten vermochte. Auch Dienstboten und die auf Stör gehenden Handwerker wurden vornehmlich mit Naturalien bezahlt. So blieb der Geldverkehr im Bergbauernhof bis weit ins 19. Jahrhundert hinein ohne grosse Bedeutung.

Auf den Merkantilismus unter Ludwig XIV folgte als Gegenströmung der Physiokratismus. Sein Begründer, François Quesnay (1694-1774), verkündete die These: "L'agriculture est la source de toute richesse de l'Etat". Die physiokratische 
Bewegung ist in Bezug auf die Landwirtschaft der Alpen deshalb von Bedeutung, weil sie zu Gründungen von naturwissenschaftlichen Organisationen führte, die sich der landwirtschaftlichen Forschung, dem Meliorationswesen und insbesondere auch der Verbreitung ihres Wissens annahmen. Für die Schweiz sind namentlich die "Oekonomische Gesellschaft von Bern" und die "Naturwissenschaftliche oder physikalische Gesellschaft in zürich" zu erwähnen. Ein Zitat aus den Schriften einer Kommission der Zürcher Gesellschaft soll deren Einsatz für die Verbesserung der Landwirtschaft belegen: Sie (die Kommission) "beratschlaget, wie die Naturlehr zu praktischem Nutzen des Landmanns angewendet werden könne, und macht Anstalten, dass das gut Gefundene dem Landmann bekannt und von diesem ausgeübt werde" (Howald, 1946). Zur gleichen Zeit erscheint auch die Schrift des zürcher Stadtarztes Johann Kaspar Hirzel mit dem Titel: "Die Wirtschaft eines philosophischen Bauern". Dieser Bauer (Kleinjogg) wird von Goethe besucht und in seinen Reiseberichten in hohen Tönen verewigt. Diese Bewegungen, in allen Kantonen verbreitet, brachten wissenschaftliche Erkenntnisse zu den Bauern. Insbesondere zeigten sie, wie Düngung, Bodenbearbeitung und Fütterung verbessert werden konnten. Damit war die Wissenschaft der modernen Agronomie und auch des modernen Meliorationswesens geboren. Letzteres ist vor allem praktiziert worden, indem grosse versumpfte und sporadisch überschwemmte Talsohlen entwässert wurden. Das diesbezüglich bekannteste Werk der damaligen Zeit ist die Linthkorrektion zwischen Walensee und Zürichsee.

\section{c) Die wirtschaftliche Not in der liberalen Epoche}

Die Tätigkeiten der von den städten ausgehenden physiokratischen Bewegungen wurden nach und nach von Organisationen der Landbevölkerung übernommen. Die Zielsetzungen blieben die gleichen, nämlich Melioration von Böden, Förderung des Unterrichtswesens und Organisation des landwirtschaftlichen Kreditwesens. Preis- und Marktpolitik gab es noch keine, weil die damalige Marktlage und der Entfernungsschutz die lokale landwirtschaftliche Produktion begünstigten.

Unter diesem Entfernungsschutz war der Ackerbau nicht nur in den Talgebieten bzw. Tiefebenen, sondern auch in den Alpengebieten sehr stark verbreitet. Dieser Situation wurde durch den Wegfall des Entfernungsschutzes ein Ende gesetzt: Dampfschiffe und Eisenbahnen brachten nun Grundnahrungsmittel

(v.a. Getreide) von Uebersee auch in unsere Bergtäler. Als "Kornkrise", die von vielen Bauernkonkursen begleitet war, ging so die Zeit zwischen 1860 und 1890 in die Agrargeschichte ein. In der Folge begann im Alpenraum und in den übrigen Gebieten eine Umstellung vom dominanten Getreidebau auf Viehwirtschaft und Milch- und Fleischproduktion. Dies waren Anfänge der noch heute andauernden "Milchkrise", die, wie noch $\mathrm{zu}$ zeigen sein wird, für die Berglandwirtschaft sehr bedeutungsvoll ist. Gleichzeitig waren aber sehr viele Leute ins "neue Paradies" Amerika ausgewandert. Manche kamen zurück, andere fanden in Amerika unter zum Teil sehr schweren Verhältnissen eine neue Heimat. 
Zur Darstellung der alpinen Landwirtschaft gehören einige Hinweise auf den sogenannten Welschlandhandel. Darunter versteht man vor allem den Export von Lebendvieh durch die Bergbauern der Nord-Schweiz auf die oberitalienischen und Tessiner Märkte wie jene von Tirano, Bergamo, Brescia und Lugano. Doch dieser Export war ein risikoreiches Geschäft. Eingehende Schilderungen dieser Märkte liefert uns Johann Andreas von Sprecher in seiner 1875 erschienenen "Kulturgeschichte der drei Bünde" (Sprecher, 1875).

Das 19. Jahrhundert ist für den Alpenraum auch die Zeit der Abholzung ganzer Hänge, um unter anderem Futterflächen zu gewinnen. Der wichtigste Auslöser war aber die grosse Nachfrage nach Holz für den Aufbau der Industrie (Fabrikbauten, Eisenbahnen, Schiffsbau, etc.). Die Flutkatastrophe von 1868, die viele Dörfer überschwemmte und ganze Täler verwüstete, strafte diesen fahrlässigen Raubbau an der Natur. Manche Bergler nahmen auch jene Flutkatastrophe zum Anlass, ihr Heil in Amerika zu suchen. In der Schweiz hatte diese Flutkatastrophe zur Folge, dass man um 1890 ein Forstgesetz erliess, das (aus heutiger sicht) den fortschrittlichen Artikel der absoluten Erhaltung der schweizerischen Waldfläche enthält.

\section{d) Krisen und Kriege zwischen 1900 und 1950}

Als Folge der "Kornkrise" entstand 1893 in der Schweiz das erste Landwirtschaftsgesetz. Es war ein Subventionsgesetz für das landwirtschaftliche Forschungs- und Unterrichtswesen. Für die Landwirtschaft in den Alpen brachte es nichts Wesentliches. Lediglich die Marktpreise für Agrarprodukte stiegen allmählich wieder an. Insbesondere erreichten die Preise während des ersten Weltkrieges sozial ungerechte Höhen. Den Bauern ging es gut, während manche landlose Arbeiterfamilie Not litt.

Die Jahre zwischen den beiden Weltkriegen waren geprägt durch dramatische Preiszusammenbrüche. In den Jahren 1921 bis 1929 bewegte sich das landwirtschaftliche Arbeitseinkommen auf einem tiefen Niveau. Mit der Weltwirtschaftskrise (1929) verschlechterte sich die Situation abermals. Besonders die Agrarpreiszusammenbrüche auf den Milch- und Viehmärkten trafen die Bergbauern hart. Die Exportmärkte versiegten, so dass in der Schweiz um 1930 erste Agrarmarktstützungen des Bundes eingeführt wurden. Doch die dreissiger Jahre waren schwierige Jahre, wohl nicht nur für die Landwirte, sondern auch für die Beschäftigten in Gewerbe und Industrie. Für viele Arbeitslose war die Nebenerwerbslandwirtschaft die Rettung vor allzu grosser Not. Während der Zeit des Zweiten Weltkrieges war die Landwirtschaft der Schweiz durch die Anbauplanung und die Lebensmittelrationierung geprägt. Ein Ergebnis davon war, dass in den Bergtälern der Ackerbau, namentlich Kartoffeln und Gerste, sich wieder stark ausdehnte. Wohl waren es harte Zeiten für die Landbewirtschafter, aber die geregelte Verteilung führte dazu, dass soziale Spannungen wie im Ersten Weltkrieg ausblieben. 
Ein Blick auf die ersten fünfzig Jahre unseres Jahrhunderts zeigt, dass sich Krisen und Kriege ablösten. Unsicherheiten prägten auch das Schicksal der Bevölkerung im alpinen Raum. In dieser Zeit weisen die Alpengebiete in Frankreich und Italien Bevölkerungsabnahmen von rund 50 bzw. $30 \%$ auf. In der Schweiz wanderten die Leute vor allem aus dem Kanton Tessin aus, während die Berggebiete der Zentral- und Ostschweiz in dieser Zeit kaum grosse Bevölkerungsverluste aufweisen.

Diese geschilderte Situation bildet fortan die Ausgangslage für eine Entwicklung der Berglandwirtschaft im Sog der modernen Wirtschaftsentwicklung. Darauf treten wir im folgenden Abschnitt ein.

\section{ZUR HEUTIGEN SITUATION DES ALPENRAUMES}

Zur Darstellung der heutigen Situation im Alpenraum werde ich zu zwei Aspekten einen Kommentar geben, nämlich (a) zu den heutigen Dorftypen und (b) zu den darin wirtschaftenden Bauernbetrieben.

\section{a) Dorftypen als Ergebnis der wirtschaftlichen Entwicklung}

Um 1950 herum waren die meisten Dörfer im Berggebiet noch Bauerndörfer. Ausnahmen bildeten einige wenige Kurorte wie St.Moritz, Davos und Zermatt. In den Bauerndörfern lebten, bezogen auf den kargen Boden, relativ viele Leute. Dann setzte im Berggebiet der Wasserkraftwerkbau und im Talgebiet ein hohes Industriewachstum ein. Es entstand eine grosse Nachfrage nach Arbeitskräften im Baugewerbe und seinem Nebengewerbe, aber auch ein Sog nach Lehrlingen in die Fabriken des Talgebietes. Viele Landwirte konnten ohne Wohnortswechsel einen anderen Beruf aufnehmen. In das Talgebiet, also in die Industriezentren, wanderten vor allem die Schulabgänger $\mathrm{ab}$. Gleichzeitig verschwanden in den Bergdörfern viele Gewerbebetriebe, weil deren Produkte jetzt billiger in den Fabriken der Industrie hergestellt wurden. Ein erster Schub war damit vollzogen. Der zweite folgte, als in den sechziger Jahren der Wintertourismus aufkam. Die davon induzierten Bau- und Holzgewerbebetriebe und das Gastgewerbe brachten viele Arbeitsplätze. Diese Entwicklung liess aber drei Arten von Bergdörfern entstehen, nämlich Industriedörfer, Tourismusdörfer und Bauerndörfer. Bekanntlich sind aber nicht alle Talschaften für den Wintersport geeignet. Wo dies nicht der Fall war, wanderten viele junge Leute gänzlich ab oder - wie typischerweise im Kt. Wallis - wurden viele zu Tagespendlern. Deren Frauen und Grossväter betreiben dann in der Regel eine kleine angestammte Nebenerwerbslandwirtschaft.

Abbildung 1 stellt für drei Gemeinden der Schweiz dar, in welchen Sektoren dort gearbeitet wird. In Davos sind gut drei Viertel aller Beschäftigten im Dienstleistungssektor tätig. Der bedeutende Tourismus führte zu einem wirtschafts- 
wachstum, das die Gemeinde zu einem regionalen Zentrum werden liess, was den dritten Sektor nocheinmal stärkte. Die Land- und Forstwirtschaft ist mit einem Anteil von knapp 4\% der Beschäftigten unbedeutend.

In der Gemeinde Visp wurde die zur Verfügung stehende elektrische Energie zum Aufbau der chemischen Industrie genutzt. Daher sind $62 \%$ aller Beschäftigten im II. Sektor tätig. Auch diese Gemeinde wurde zum regionalen Zentrum, was den Anteil der Beschäftigten im Dienstleistungssektor beeinflusst. Die Land- und Forstwirtschaft trägt wiederum nur einen geringen Anteil zur Beschäftigung bei.

In einer ganz anderen Situation befindet sich Vrin. Von den nur 96 Beschäftigten in dieser Gemeinde sind 57 in der Landund Forstwirtschaft tätig. Die Beschäftigten im zweiten und dritten Wirtschaftssektor halten sich etwa die Waage. Diese Gemeinde ist klein geblieben, und ihre Wirtschaft hängt fast ausschliesslich von der Landwirtschaft $a b$. Veränderungen in der Mechanisierung und der damit verbundene strukturwandel wirken sich daher stark auf die dörfliche Wirtschaft und somit auch auf die Bevölkerungszahl aus.

\section{Abb. 1: Sektorielle Verteilung der erwerbsfähigen Bevölke- rung}

Davos

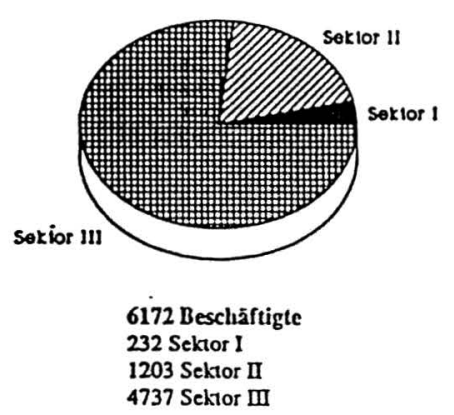

Visp

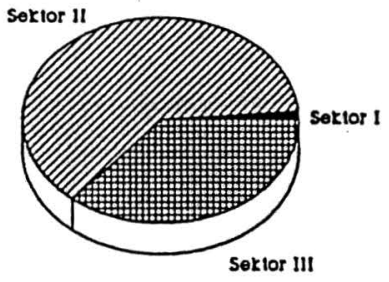

5229 Beschäftigte

88 Sektor I

3752 Sekior II

2171 Sektor III

\section{Vrin}

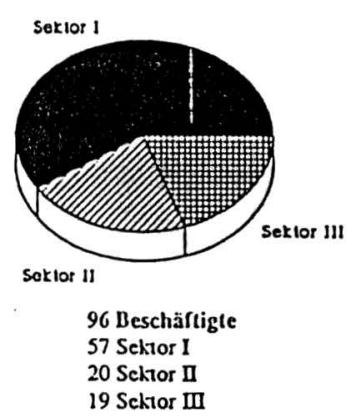

Quelle: Eidg. Betriebszählung 1985 
Aus der Einsicht in die Notwendigkeit eines sozio-ökonomischen Gleichgewichtes in einem Bergdorf entwickelten wir unser Dorfmodell. Wir nennen es unser Modell, weil im Zentrum der einzelne Landwirt steht, der die in der Regel einzige ortsgebundene Tätigkeit im Dorfe ausübt. Ergebnis seiner Tätigkeit ist die Kulturlandschaft. Des weiteren gehen wir nun davon aus, dass der Einzellandwirt ein wirtschaftliches und soziales Umfeld braucht. Ohne dieses würde auch er aus Gründen geistiger und ökonomischer Isolation abwandern. Abbildung 2 dürfte im übrigen für sich sprechen: Unmittelbares Umfeld für den Landwirt sind Betriebsgemeinschaften (z.B. Alpen), Genossenschaften zur Produkteverarbeitung und Berufsvereine. Dann folgt das Umfeld der dörflichen Funktionen, die ein Bewohner grundsätzlich täglich in Anspruch nehmen kann. Es sind dies kulturelle, gesellschaftliche, wirtschaftliche, schulische und religiöse Einrichtungen. Schliesslich enthält Abbildung 2 das Feld der regionalen Funktionen. Auch diese sind von existentieller Bedeutung für ein Bergdorf und seine Landwirte.

\section{Abb. 2: Abhängigkeiten zwischen den Funktionen einer Dorfge- meinschaft}

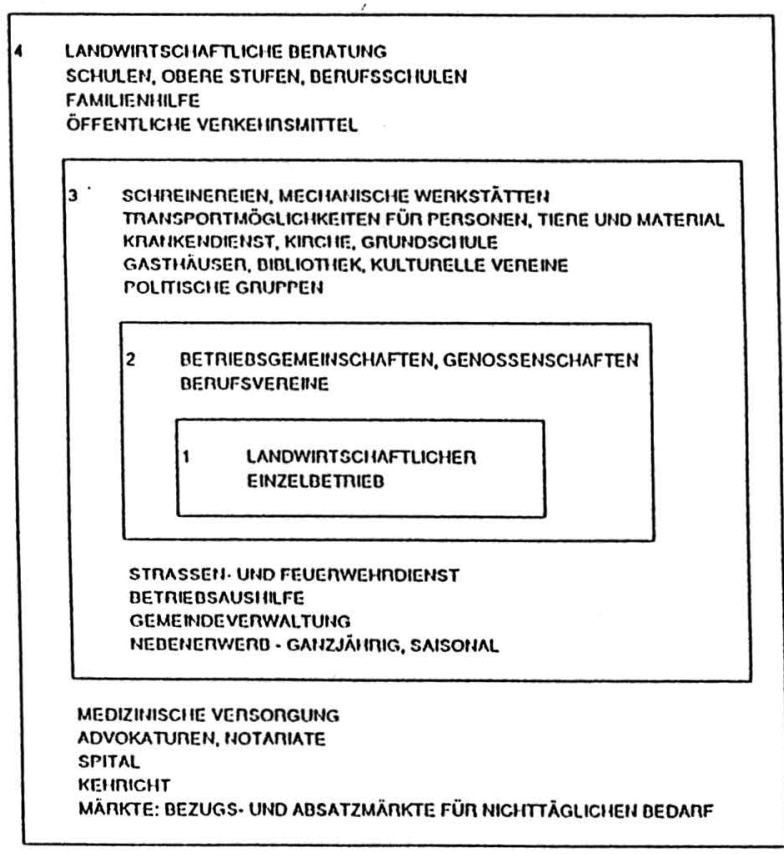

1. Ebene Einzelbetrieb

2. Innerlandwirtschalliche Funktionen
3. Funktionen innerhalb der Dorfgemeinschaft

4. Regionale Funktionen

Mit dem Konzept des Dorfmodelles kann man zwei Arten von Berechnungen vornehmen. Erstens lässt sich feststellen, was in einem Dorf zu seinem Funktionieren fehlt bzw. Wo Investitionen von existentieller Notwendigkeit sind. Zweitens lässt sich berechnen, wie gross eine minimale Dorfgrösse sein sollte, damit die öffentlichen (Schulen, etc.) und die privaten (Läden, Restaurants, etc.) Einrichtungen existieren 
können. Nach unseren Berechnungen und Analysen in bündnerischen Gemeinden beträgt die Minimalgrösse ca. 500 Einwohner (Rieder, 1984).

\section{b) Bauernbetriebe in Bergdörfern}

Es kann nicht darum gehen, die Strukturen der Landwirtschaftsbetriebe umfassend darzustellen. Vielmehr beschränke ich mich der Anschaulichkeit wegen auf einige Hinweise. Wir halten verallgemeinernd fest:

Ueberall stellt man anhaltende Betriebsvergrösserungen fest. Ueberall hat es Haupterwerbs- und Nebenerwerbsbetriebe. Je bäuerlicher das Dorf jeweils ist, umso weniger Nebenerwerbsbetriebe sind vorhanden. Aber auch Dörfer mit starker Wirtschaftsentwicklung, z.B. Davos, weisen wenig Nebenerwerbsbetriebe auf. Dazwischen gibt es eine Art "mittlere" Dörfer mit dem relativ grössten Anteil an Nebenerwerbsbetrieben.

\section{Abb. 3: Landwirtschaftliche Betriebsstrukturen in Safien}

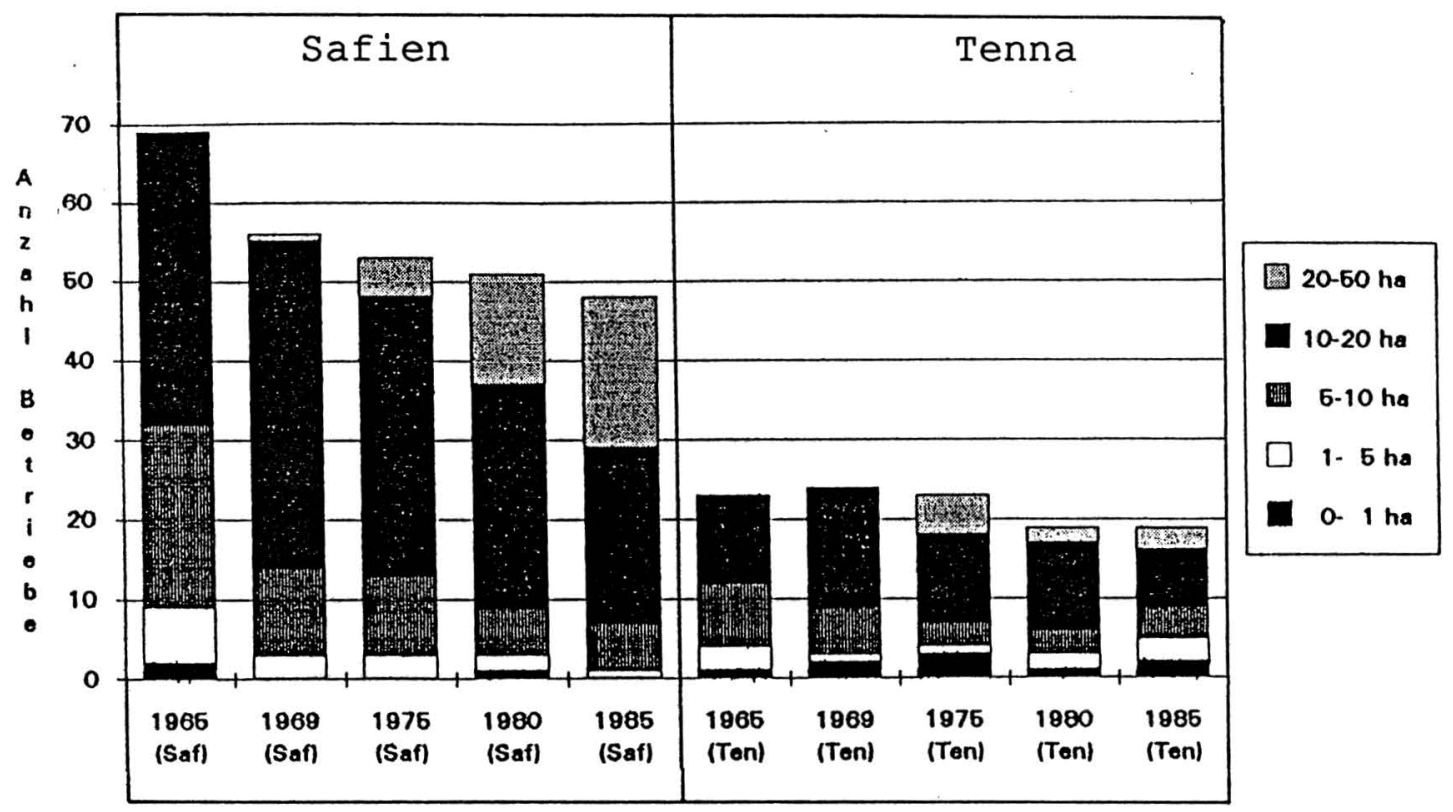

Quelle: BfS: Eidg. Betriebszählungen

Zuerwerb unter Haupterwerbsbetrieben gibt es vor allem dort, wo Ski-Anlagen bestehen. Die Betriebsgrössen verändern sich eindeutig umso schneller, je grösser das Wachstum der übrigen Wirtschaft ist. Die Einkommenslage der Bergbauern ist dank staatlicher Stützung mittelmässig. Abbildung 3 zeigt am Beispiel der zwei Gemeinden im Safiental, wie unterschiedlich Agrarstrukturveränderungen sein können. Tenna weist mit grösseren Betrieben sehr stabile Verhältnisse auf, während in Safien der Prozess voll im Gange ist, sich aber auch ei- 
ner stabileren Situation mit Betrieben mit über 20 Hektaren Kulturfläche annähert.

\section{EIN KOMMENTAR ZUR HEUTIGEN AGRARPOLITIK ZUGUNSTEN DER BERGLANDWIRTSCHAFT}

Bevor zukünftige Konzepte angesprochen werden können, muss die aktuelle Agrarpolitik zugunsten der Berglandwirtschaft dargestellt werden. Es soll ja sichtbar werden, wo diese allenfalls abzuändern bzw. zu erweitern wäre.

Bereits im Bundesgesetz über die Förderung der Landwirtschaft und Erhaltung des Bauernstandes vom 3. Oktober 1951 (Landwirtschaftsgesetz, LwG) ist festgehalten, dass die erschwerten Produktions- und Lebensbedingungen in den Berggebieten bei der Durchführung des Gesetzes zu berücksichtigen seien (Art.2, Abs.1). Dieser Grundsatz wurde aber anfangs von Art.29 des LwG über die Gewährung kostendeckender Preise für Inlandprodukte überschattet. Erst durch den Druck der sich vergrössernden Einkommensdisparitäten zwischen Tal- und Berggebiet kam es Ende der 50er/anfangs der 60er Jahre zur Realisierung differenzierter Massnahmen zugunsten der Berglandwirtschaft. Diese haben zum Teil sozialpolitischen Charakter, bilden zum andern aber auch die Grundlage für direktwirksame marktwirtschaftliche Instrumentarien.

Gleichzeitig mit diesen ersten Differenzierungen wurden 1958 die drei Bergzonen nach Viehwirtschaftskataster geschaffen. Die Zonenregelung wurde allmählich zu einem feinstufigen Zonensystem ausgebaut: Voralpine Hügelzone (1971), Bergzone IV (1980), im Talgebiet die ackerbaulich relevante Ueberganszone (1977) und die erweiterte Uebergangszone (1982). Die Zoneneinteilung war eine wichtige Grundlage, um agrarpolitische Massnahmen überhaupt differenzieren zu können.

In der Folge sind die berggebietsspezifischen Gesetze und Direktzahlungen zusammengestellt:

\section{Viehabsatzgesetz:}

Bundesgesetz über die Förderung des Absatzes von Zuchtund Nutzvieh, von Pferden und Schafwolle, vom 15. Juni 1962. Ab 1978 Viehabsatzgesetz.

Dieses Gesetz bildet die Rechtgrundlage für die marktpolitischen Instrumentarien wie Ausmerzbeiträge, Entlastungskäufe und Exportbeiträge von Zucht- und Nutzvieh aus dem Berggebiet. Die Möglichkeit von Exportbeiträgen bestand jedoch bereits seit 1953 in der Allgemeinen Landwirtschaftsverordnung vom 21. Dezember 1953.

\section{Kostenbeitragsgesetz}

Bundesgesetz über Kostenbeiträge an Viehhalter im Berggebiet und in der voralpinen Hügelzone:

Kostenbeiträge wurden erstmals 1959 im Milchwirtschaftsbeschluss eingeführt. 1964 wurde ein eigenes Gesetz geschaffen, das 1971 ausgedehnt und am 28. Juni 1974 neu konzipiert wurde. Die letzte Teilrevision fand 1982 statt. 
ziel: Abgeltung der höheren Produktionskosten im Berggebiet und der voralpinen Hügelzone.

\section{Flächenbeitragsgesetz (inkl. Sömmerungsbeiträge)}

Bundesgesetz über Bewirtschaftungsbeiträge an die Landwirtschaft mit erschwerten Produktionsbedingungen, vom 14. Dezember 1979.

ziel: Erhaltung der Landwirtschaft in Lagen mit erschwerten Produktionsbedingungen und Sicherung der Einkommen durch Abgeltung des Mehraufwandes.

Die Sicherstellung von Schutz und Pflege von Grenzertragsland über Beitragszahlungen und über die Verpflichtung der Grundeigentümer, die Bewirtschaftung und Pflege von Brachland zu dulden (Duldungspflicht).

\section{Zusatzkontingente}

Milchwirtschaftsbeschluss vom 7 . Oktober 1977; Art.7

Ziel: Förderung des Rinderkaufs aus dem Berggebiet durch Erhöhung der Milchkontingente für Käufer von Rindern aus dem Berggebiet.

\section{Tierhalterbeiträge}

Landwirtschaftsgesetz, 3. Oktober 1951, Art.19c; Verordnung über Beiträge an Tierhalter vom 14. März 1988. Diese sind nicht spezifisch auf das Berggebiet beschränkt.

ziel: Abgeltung der erhöhten Produktionskosten für Tierhalter, die kleinere bis mittlere Betriebe bewirtschaften.

6. Vorgesehene Betriebsbeiträge

LwG, Art. 31b

Ziel: Struktur- und produktionsneutrale Beiträge zur Sicherung der Einkommen. Sie sollen einzig an ökologische Bedingungen gebunden sein.

Die sechs aufgeführten Gesetze sind zeitlich gestaffelt eingeführt worden. Daraus erklären sich ihre unterschiedlichen ökonomischen Eigenschaften. Das Viehabsatzgesetz gab ab 1952 als erstes die Möglichkeit, auf den Märkten zu intervenieren. Der beabsichtigte Empfänger der Subvention spürte diese nur indirekt, indem er höhere Preise für seine Tiere erzielte.

$\mathrm{Ab} 1959$ wurden Kostenbeiträge ausgerichtet. Damit erfolgte erstmals eine Zahlung, die direkt den Bauern ins Haus geschickt wurde. Das Bemessungskriterium war nicht mehr das Produkt auf dem Markt, sondern die Produktionsfaktoren auf dem Betrieb, nämlich seine Tiere, auf Grossvieheinheiten umgerechnet. Bezüglich Bemessungsgrösse ist also ein erster Schritt weg vom Produkt, hin zu den Produktionsfaktoren erfolgt. 
Das Flächenbeitragsgesetz (1980) geht noch einen Schritt weiter, indem Beiträge pro Fläche ausgerichtet werden, unabhängig davon, was darauf produziert wird. Die Flächen müssen nur Bedingungen bezüglich Steilheit und geordneter Nutzung erfüllen. Vor allem soll damit der Ausdehnung von Brachland einhaltgeboten werden.

Bei den Tierhalterbeiträgen ging man 1988 insofern noch einen Schritt weiter, als dass jedem Bauer innerhalb unterer und oberer Grenzen der gleiche Betrag, z.z. 4500.- Fr., zusteht, sofern er Tiere hält bzw. ein Bauer ist.

Die Zusatzkontingente sind spezifisch in der Absicht eingeführt worden, den Tallandwirten einen ökonomischen Anreiz zum Zukauf von Tieren aus dem Berggebiet zu geben. So entspricht dieses Gesetz eher dem Charakter des Viehabsatzgesetzes.

Die zeitliche Abfolge der Gesetze spiegelt die zunehmende Marktenge und die sich verschlechternde innerlandwirtschaftliche Einkommensverteilung wider. Es erwies sich sowohl als ökonomisch sinnvoll wie auch als wirtschaftspolitisch gerecht, Massnahmen zunehmend produktionsneutraler und dafür einkommensorientierter zu gestalten. Mit der zur zeit vorgesehenen Ergänzung des Landwirtschaftsgesetzes mit Art.31a und $\mathrm{b}$ sollen allgemeine Direktzahlungen auch für Tallandwirte möglich werden. Diese Entwicklung ist eine notwendige Folge bei Einkommenssicherung auf überlasteten Agrarmärkten.

Abb. 4: Uebersicht über Direktzahlungen für die Berglandwirtschaft in der Schweiz

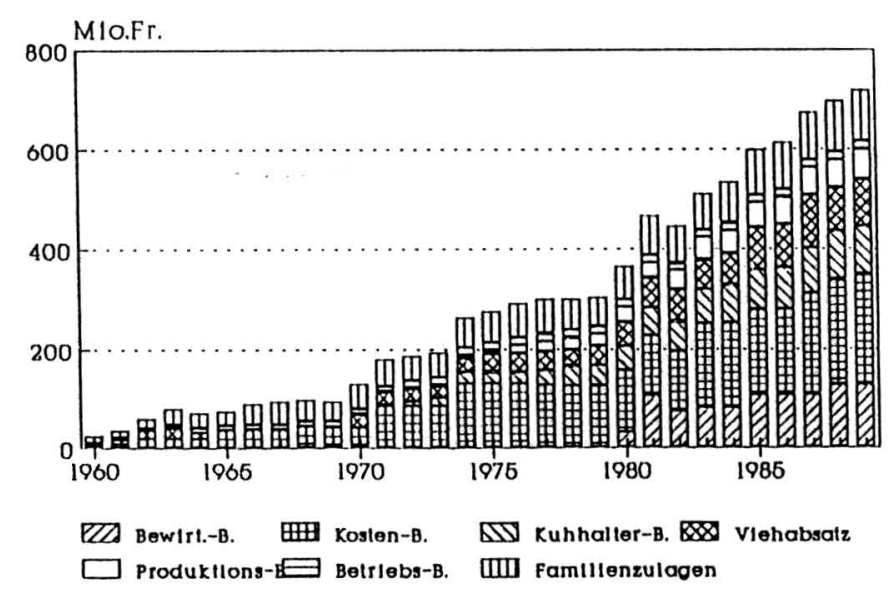

Quelle: Buergi (1989) 
Unter diesem Aspekt ist nun zweigeleisig zu fahren. Es sind nämlich sowohl die Sicht des Einzelbauern als Kleinunternehmer als auch die öffentliche Sicht bzw. die öffentlichen Funktionen miteinzubeziehen. Vorerst einige Aspekte zum Einzelbauern.

Um das Verhalten des Einzelbauern im landwirtschaftlich schwierig zu nutzenden Berggebiet zu verstehen, eignet sich ein Zielsystem mit den Zielen (a) befriedigende Einkommen, (b) normale Arbeitsbelastung (c) soziale Integration und (d) Sinnerfüllung in der Arbeit. Wie Abbildung 5 zeigt, stehen diese ziele in einer interdependenten Beziehung zueinander. Die Aussage dazu lautet: Der Berglandwirt verhält sich zu jedem Zeitpunkt so, dass er durch Veränderungen jenes ziel besser zu erreichen sucht, das für ihn subjektiv am wenigsten erreicht ist.

\section{Abb. 5: Zielsystem des Einzellandwirtes}

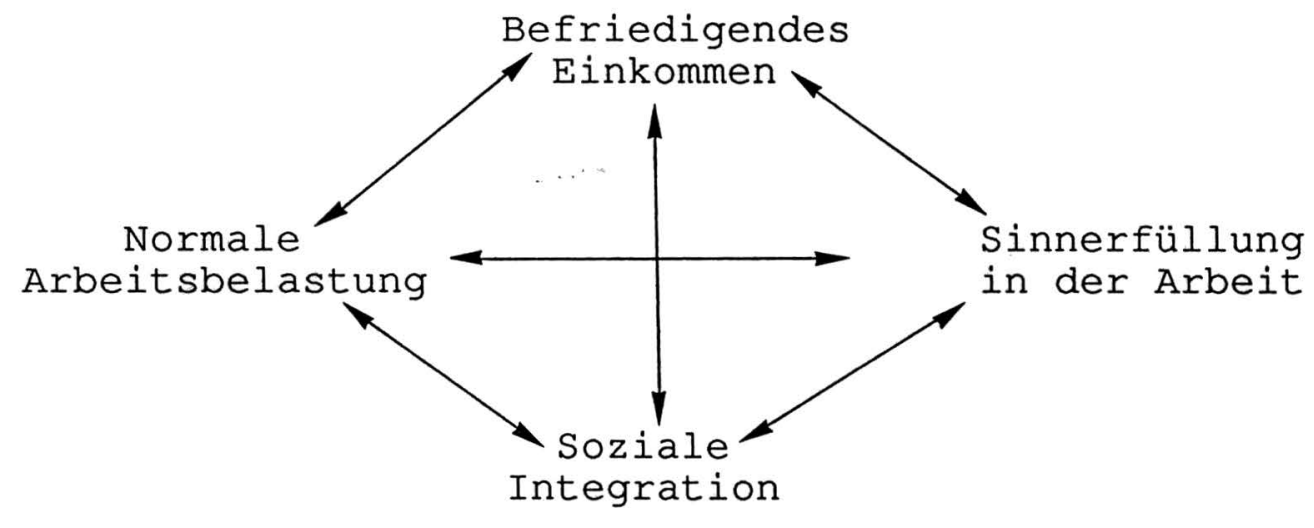

Die Relevanz für den Bergbauern ist deshalb so gross, weil die Arbeitsbelastung zu Zeiten ausschliesslicher Handarbeit (Heueintragen, Mistverteilen, keine Güterwege, etc.) äusserst gross waren. Subjektiv gesehen wurden bei aufkommender Mechanisierung äusserst schnell viele Maschinen angeschafft, obwohl es "arme Bergbauern" waren. Abbildung 6 zeigt, wie schnell sich im Kanton Graubünden die Mechanisierungsstufen wandelten. Aus rein betriebswirtschaftlicher sicht ist oft eine Uebermechanisierung festzustellen; aus der Sicht des zielsystems ist das Verhalten rational erklärbar. Auch Soziale Integration ist nur möglich, wenn die Einkommen befriedigend sind und die Arbeitsbelastung normal ist. 
Abb. 6: Flächennutzung nach Mechanisierungsstufen in Graubünden

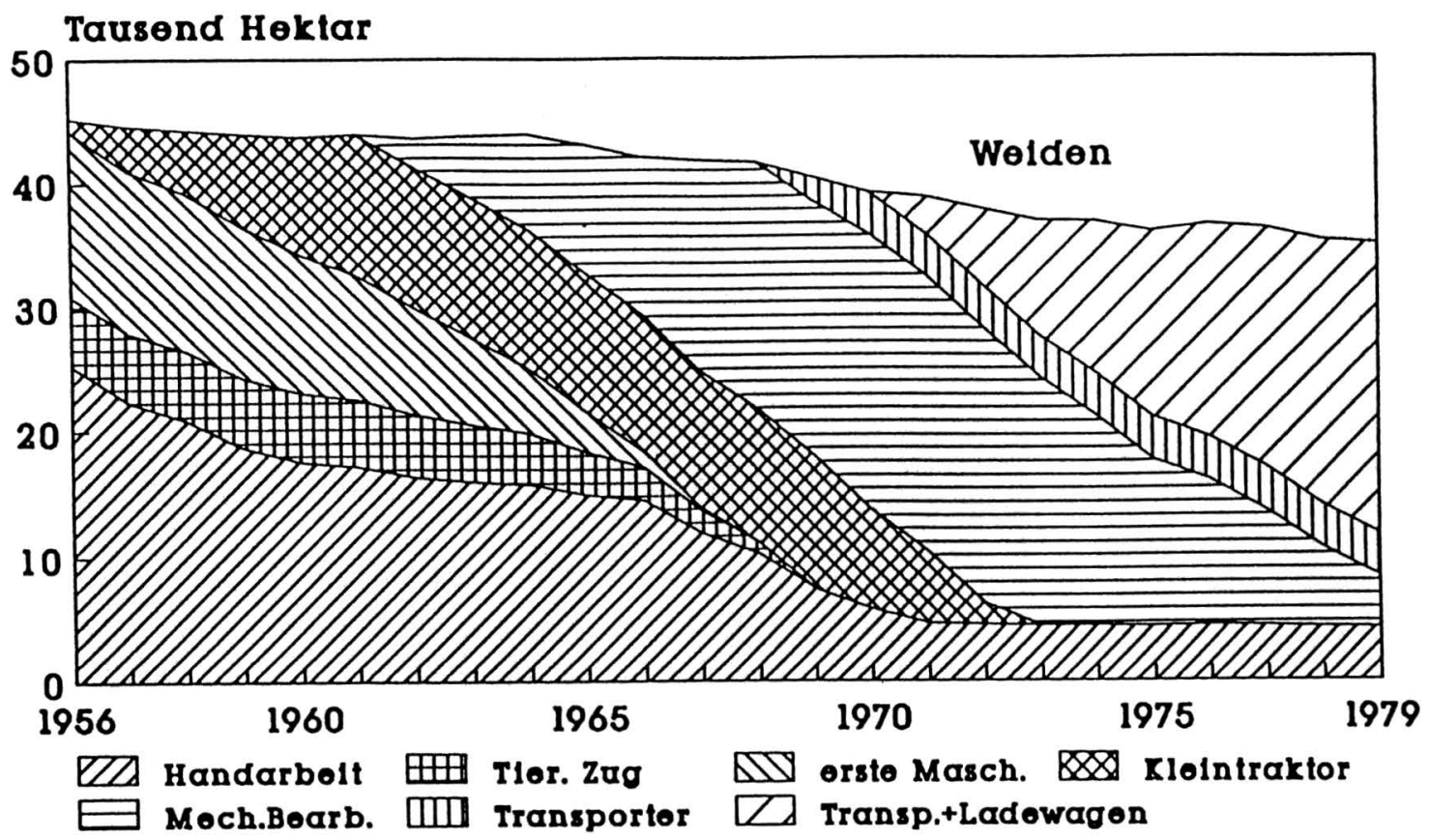

Quelle: Bernegger (1985)

Gleiche Ursachen wie die theoretische Uebermechanisierung hat die oft festzustellende Polarisierung der Landnutzung: Mechanisch gut bearbeitbares Land wird übernutzt und überdüngt, so dass der Pflanzenbestand einseitig wird.

Anderseits entsteht dort Brachland, wo Zufahrtswege fehlen oder das Land zu stark kupiert ist. Letzteres von Hand $z u$ bewirtschaften ist $\mathrm{zu}$ aufwendig, um davon wirtschaftlich existieren zu können. Die Einsicht in die geschilderten Zusammenhänge ist bei den Söhnen der bäuerlichen Väter in den Dörfern und bei Auswärtigen und Feriengästen nicht immer vorhanden. Es zeigt sich etwa darin, dass Touristen im Berggebiet nicht das Normale der bäuerlichen Tätigkeit fotografieren, sondern das Archaische, was schon längst ausser Nutzung ist. Soweit zum individuellen Zielsystem.

Das öffentliche Zielsystem enthält andere nachgefragte Funktionen, so die Erhaltung der Kulturlandschaft, die dezentrale Besiedlung, keine Umweltbelastung und blumenreiche Wiesen u.a.m. Diese Funktionen fallen bei Verhalten nach individuellem zielsystem nicht mehr alle in nachgefragtem Ausmass an. In dieser mangelnden Kongruenz der ziele liegt ein Problem begraben. Also stellt sich die Frage, welche Politik bzw. welche Massnahmenpakete zu einer möglichst konfliktfreien maximalen Erreichung sowohl individueller als auch öffentlicher Ziele führen. 

Agrarpolitik der Schweiz ohne EG- und GATT-Aspekte

\section{a) Probleme der bisherigen Agrarpolitik}

Die Agrarpolitik basiert seit der Einführung des Landwirtschaftsgesetzes im Jahre 1951 faktisch auf dem Grundsatz produktionsdeckender Preise mittels inländischer Preisstützung und Aussenhandelsschutz. Die Preispolitik ist somit in der Schweiz das wichtigste agrarpolitische Instrument. Sie beinhaltet den zentralen Zielkonflikt, dass über die Produktepreise sowohl Angebotssteuerung als auch Einkommenspolitik betrieben wird. Die Politik der über dem Marktgleichgewicht festgelegten Preise führte einerseits zu Marktüberschüssen und steigenden Preisunterschieden zum umliegenden Ausland und anderseits zu ungewollten Einkommensparitäten innerhalb der Landwirtschaft, die man im Voralpen- und Berggebiet schon heute mit zusätzlichen Direktzahlungen auszugleichen versucht. Im Laufe der Zeit kam zu den zwei genannten Problemen ein dritter Problemkreis hinzu, nämlich die ökologischen Gefahren durch eine zu hohe Produktionsintensität einerseits und das Auftreten von Brachland in abgelegenen Gebieten anderseits.

\section{b) Die Erweiterung des agrarpolitischen Instrumentariums mit einkommens- und umweltpolitischen Massnahmen}

Nach Abbildung 7 wird die schweizerische Agrarpolitik in die vier Bereiche Sozialpolitik, Strukturpolitik, Einkommenspolitik und Umweltpolitik eingeteilt:

Soziale Massnahmen erfüllen in der Landwirtschaft die gleichen Funktionen wie in der übrigen Wirtschaft und bezwecken die Reduktion von Soziallasten. Im Zusammenhang dieser Ausführungen wird daher nicht weiter darauf eingegangen.

Die Strukturpolitik dient der Verbesserung der Produktionsgrundlagen. Sie ist längerfristig ausgelegt und bildet Anreize zur Realisierung von effizient $\mathrm{zu}$ bewirtschaftenden und lebensfähigen Betriebseinheiten. Wichtigstes strukturpolitisches Instrument sind in der Schweiz zinsgünstige Investitionskredite und Beiträge für Meliorationen und Stallbauten.

Die Einkommenspolitik besteht aus preispolitischen Instrumenten und den Direktzahlungen. Die Preispolitik beruht auf inländischen Preisfestlegungen, staatlichen Abnahmegarantien und dem Aussenhandelsschutz. Direktzahlungen haben zum Teil einkommenspolitischen und zum Teil umweltpolitischen Charakter. Auf die verschiedenen Formen von z.T. neuen Direktzahlungen und ihre spezifischen Wirkungsweisen wird anschliessend bei Diskussionen der Umweltpolitik eingegangen.

Die Instrumente der Umweltpolitik bestehen aus Direktzahlungen und Produktionsauflagen. 


\section{Abb. 7: Bereiche der Agrarpolitik und landwirtschaftsbezoge- nen Umweltpolitik}

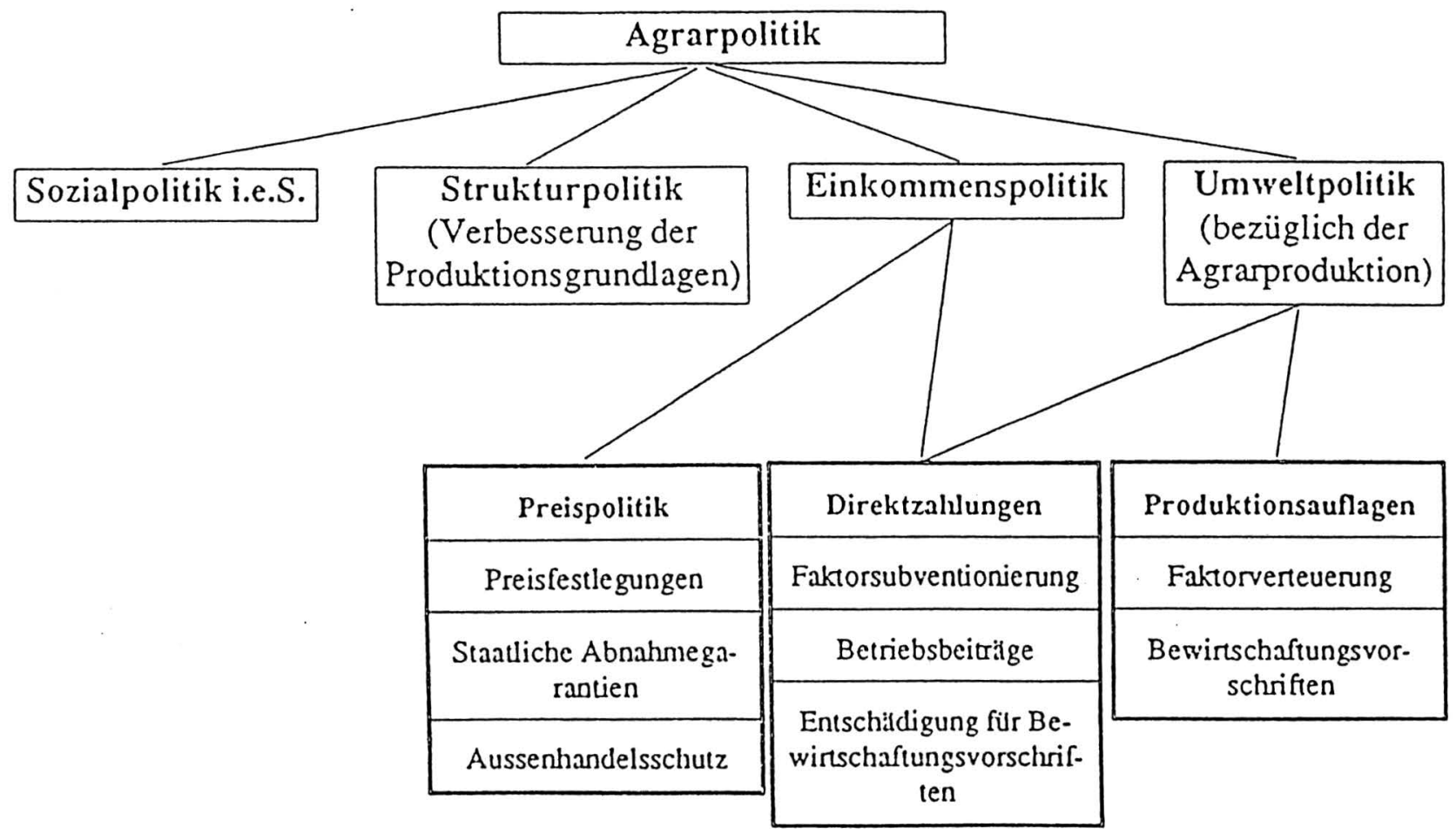

Umweltgefährdungen sollen vermieden werden über Faktorverteuerungsmassnahmen, -subventionierungen, Betriebsbeiträge und Bewirtschaftungsvorschriften. Faktorverteuerungen bezwecken einen geringeren Einsatz der schädigenden Produktionsfaktoren. In der Schweiz wird eine solche Lenkungsabgabe vor allem für Dünger und Pflanzenschutzmittel diskutiert, um den Einsatz dieser Hilfsstoffe auf ein umweltverträgliches Mass zu reduzieren.

Unter dem Begriff Faktorsubventionierung versteht man an Produktionsfaktoren gebundene Direktzahlungen. Damit werden die Kosten der subventionierten Faktoren gesenkt. Dadurch entsteht sowohl ein ökonomischer Anreiz zu seiner Nutzung als auch ein positiver Einkommenseffekt. Faktorsubventionen sind somit nicht produktionsmengenneutral. Durch flächengebundene Subventionen soll beispielsweise im Berggebiet die Nutzung von Grenzertragsböden gefördert werden (Flächenbeiträge).

Betriebsbeiträge, wie sie im neuen Art.31a des Landwirtschaftsgesetzes vorgesehen sind, sind möglichst produktionsneutrale und strukturneutrale Direktzahlungen. Sie sollen die Einkommen aller Betriebe einer genau definierten Gruppe von Landwirten um einen absolut gleich hohen Beitrag erhöhen. Bezügereinheit ist der einzelne landwirtschaftliche Betrieb bzw. der Betriebsleiter.

Bewirtschaftungsvorschriften in Form von Geboten und Verboten sind anzuwenden, wenn durch die landwirtschaftliche Produktion irreversible Gefahren oder Schäden drohen. 
Unabhängig von GATT und EG kann zusammenfassend festgehalten werden, dass konzeptionell die heutige bzw. die sich anbahnende ergänzende umwelt- und einkommensbezogene Agrarpolitik agrarökonomisch richtig angelegt ist. Ob die Höhe der Beiträge und der Preise zufriedenstellend ist, ist letztlich eine politische Frage. Eindeutig zu geringe Summen stehen für Meliorationen, Weg- und Stallbauten zur Verfügung. Die kantonalen Meliorationsämter weisen hiefür lange Wartelisten auf. Scheinbar besteht bei den Bauern das Verlangen nach Strukturverbesserungen.

\section{Zur Zukunft unter GATT- und EG-Sichtweise}

\section{a) Entwicklungen auf den Weltagrarmärkten - Zur Notwendig- keit von GATT-Agrarverhandlungen}

Der Welthandel mit Agrarprodukten hat sich im Zeitablauf infolge des Fortschritts im Transportwesen und in der Lagerhaltung vom kolonialen Gewürzhandel zu einem Handel mit fast allen landwirtschaftlichen Erzeugnissen entwickelt. Seit dem letzten Weltkrieg hat das Volumen des internationalen Agrarhandels besonders stark zugenommen, allein zwischen 1963 und 1983 erfolgte ein Anstieg um rund 90\%. Gleichzeitig ist aber das gesamte Welthandelsvolumen um rund $180 \%$ gestiegen, so dass der Anteil des Weltagrarhandels am gesamten Welthandel in dieser Zeitspanne von rund $27 \%$ auf $15 \%$ gesunken ist.

Wichtigste Akteure des internationalen Agrarhandels sind die Industrieländer. Fast die Hälfte des Handels wickelt sich innerhalb dieser Gruppe ab. Ein weiteres Drittel des Agrarhandels erfolgt zwischen Industrie- und Entwicklungsländern und rund $10 \%$ zwischen Entwicklungsländern.

In den letzten Jahrzehnten ist das Angebot auf den Weltagrarmärkten stärker als die Nachfrage angestiegen, was tendenziell zu sinkenden Preisen führte. Es gibt auf dem Weltagrarhandel dominante und abhängige Partner. Die EG und die USA bestimmen mit ihrer Politik der Exportsubventionierung die Lage auf dem Weltmarkt. Das Abschöpfungssystem der EG führte $z u$ Angebotsausdehnungen. In der Folge gingen ihre Einfuhren zurück. Die EG wurde sogar zu einem grossen Anbieter auf dem Weltmarkt. Deswegen sanken die Weltagrarpreise. Als Reaktion darauf mussten die USA ebenfalls ihre Exportsubventionen erhöhen, denn auch sie betreiben USAinternen Agrarschutz zugunsten ihrer Farmer.

Tiefe Weltagrarmarktpreise beeinflussen insbesondere die Politik der Entwicklungsländer. Um die Versorgung der Grossstädte sicherzustellen, importieren die meisten Regierungen der Entwicklungsländer preisgünstige Nahrungsmittel. Dadurch überträgt sich das tiefe Weltmarktpreisniveau ins Innere der Länder, wodurch der Anreiz zur Steigerung der inländischen Nahrungsmittelproduktion genommen wird. Oftmals wird die Produktion von Exportprodukten sogar erzwungen, um so mittels Exporten, vor allem von Kaffee, die notwendigen Devisen für den Importbedarf zu erwirtschaften. 
Von den günstigen Weltmarktpreisen für Agrargüter profitieren vor allem die importierenden kleinen Industrieländer. So stehen der Schweiz stets beliebige Importmengen zu tiefen Preisen zur Verfügung. Durch Einfuhrabgaben wird zudem ein beachtlicher Teil des inländischen Agrarschutzes finanziert. Auch die Zentralverwaltungswirtschaften vergrösserten in jüngster Zeit ihre Importe. Es fehlte offenbar der ökonomische Anreiz, um Erträge zu steigern und die Waren effizient $\mathrm{zu}$ lagern und zu transportieren.

Die gesamthaft unbefriedigende Situation führte im Rahmen der Uruguay-Runde zur erstmaligen Aufnahme der Agrarfrage in GATT-Verhandlungen. Ziel dieser Verhandlungen ist es, die Verzerrungen des internationalen Agrarhandels in den einzelnen Ländern abzubauen und auch hier den freien Handel zu fördern.

Bei den Schlussverhandlungen der Uruguay-Runde im Dezember 1990 in Brüssel konnte man sich erwartungsgemäss nicht auf einen einheitlichen Abbau der Agrarsubventionen einigen. Während die USA und die CAIRNS-Gruppe (kleine Agrar-Exporteure) einen übersetzten Abbau der Stützungsmassnahmen von rund 70\% verlangten, blieb die EG bei ihrer offerte einer Kürzung um 30\%. Das Angebot der Schweiz in dieser Runde sah eine Reduktion der inneren Stützung um 20\% und eine Reduktion der Budgetausgaben für Exportsubventionen um $30 \%$ vor.

Im Januar 1991 wurden die Verhandlungen in Genf wiederaufgenommen. Mit grosser Wahrscheinlichkeit dürfte nach und nach der EG-Vorschlag zum Handlungsergebnis werden. Für die Schweiz ist absehbar, dass das heutige starre system des Agrarhandelsschutzes und der Mengenbeschränkungen gelockert werden muss. Doch dürften diese Einflüsse auf schweizerische Art, von der nachfolgend noch die Rede ist, gebremst werden.

\section{b) EWR und EG-Anschluss}

Die bestens bekannten EWR-Verhandlungen haben auf die Agrarpolitik der Schweiz einen geringen Einfluss. Die Einflüsse wären allenfalls indirekt über den Arbeitsmarkt und durch Liberalisierungen im Produktionsmittelhandel und der diesbezüglichen Industrie spürbar.

Ein EG-Anschluss ist unseres Erachtens für die nächsten zehn Jahre sehr unwahrscheinlich. Zu viele nicht-landwitschaftliche Kreise werden nebst den Bauern gegen einen Vollbeitritt sein.

Für unsere Betrachtungen hier ist relevant, dass wir unseren Ueberlegungen - ob über GATT, EWR oder EG - einen graduellen Abbau des gesamten produktbezogenen Agrarschutzes und der produktbezogenen Exportsubventionen unterstellen, dass wir aber den Spielraum für andere, d.h. produktunabhängige Agrar-, Regional- und Umweltschutz-Massnahmen offen behalten. Durch den produktbezogenen Abbau des Agrarschutzes wer- 
den entsprechend besonders der Milch- und Getreidemarkt betroffen sein. Der Weg dazu führt nach GATT-Vorschlägen über eine Tarifizierung, d.h. Umwandlung aller ImportschutzMassnahmen in Tarife (Wertzölle) sowie einer Trennung der Preis- und Einkommenspolitik.

\section{c) Ein Szenario zu Tarifizierung und Direktzahlungen}

Diese GATT bzw. GATT-EG-Vorschläge würden für die Schweiz folgendes bedeuten (nach GATT-Verhandlungspapieren):

Phase 1: Tarifizierung

- Festlegen der Stützungspreise (PSE)

- Berechnung der Tarife (Wertzölle)

- Berechnung der Ertragsausfälle und Ersatz durch Direktzahlungen (soweit möglich finanziert durch Tarife)

Phase 2: Abbau der Tarife (über 10 Jahre)

- Erschliessung neuer Finanzierungsquellen für den Agrarschutz (Sinkende Tarifeinahmen)

- Ausbau der Direktzahlungen in Rahmen der Einkommenspolitik

Phase 3: strukturanpassungen

- Beiträge zur Milderung der Folgen des Strukturwandels (ausserhalb GATT bzw. EG-Abmachungen)

- Faktorstillegungen

Phase 4: Konsolidierung des Agrarschutzsystems

- Ausbau der Direktzahlungen für:

- Allgemeine Dienstleistungen

- Natur und Umweltschutz

- Hilfe bei Naturkatastrophen

- Ernteversicherungen

- Regionalentwicklung

- Einkommenssicherungsprogramme

Die in Phase 1 notwendigen Direktzahlungen sollen sich noch möglichst weitgehend aus den Zolleinnahmen auf Importen decken lassen. Sie sollen kurzfristig die durch Tarifizierung auf der Basis der heutigen Marktpreise entstehenden Erlösminderungen der Produzenten ausgleichen. 
Abb. 8: Entwicklung des Agrarschutzes unter der Annahme, dass die Tarife rascher gesenkt werden als der interne Agrarschutz (über 10 Jahre, in Mrd. Franken $\mathrm{zu}$ realen Werten)

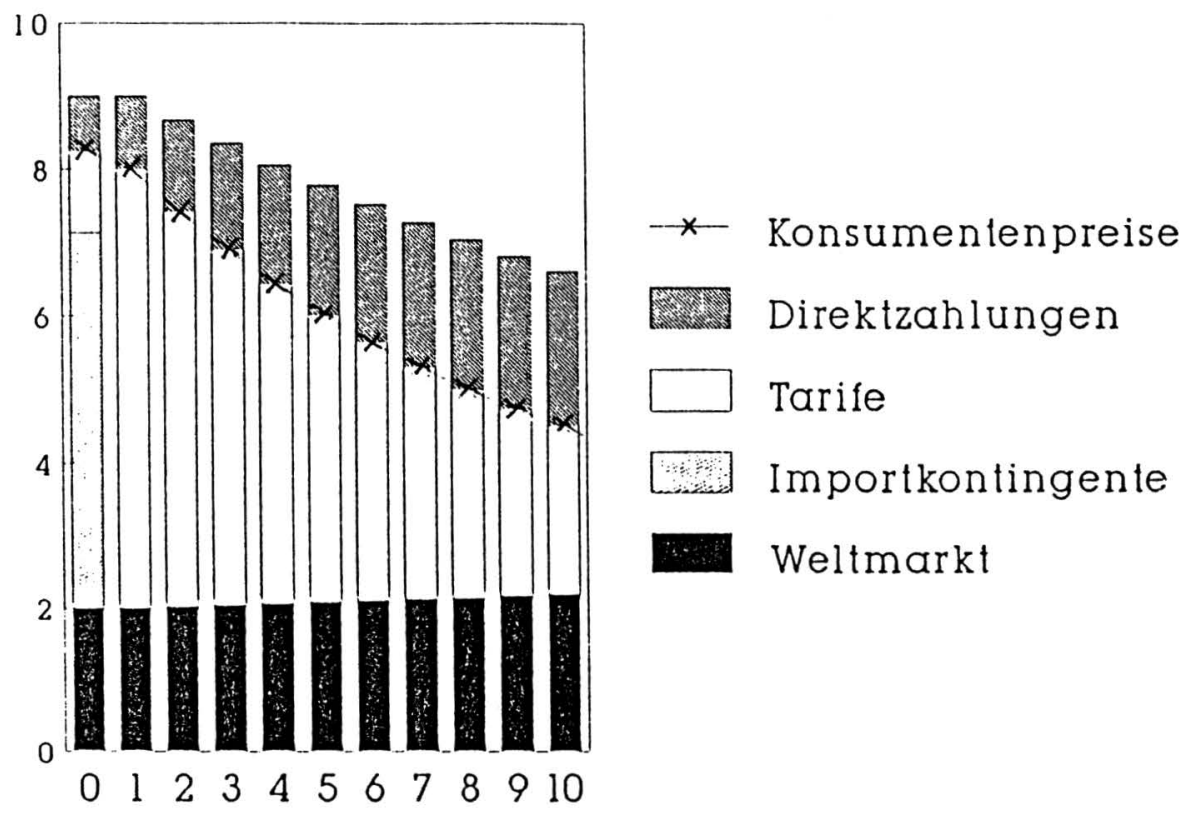

Die Phasen 2-4 sind gekennzeichnet durch eine grundsätzliche Umorientierung des Agrarschutzsystems. Eine 'progressive' und 'substantielle' Senkung des Agrarschutzes setzt voraus, dass die notwendige Strukturanpassung durch geeignete Massnahmen gesteuert werden kann. Die Senkung der Tarife schlägt dort besonders stark auf die landwirtschaftlichen Preise durch, wo auf ein hohes Agrarpreisniveau tarifiziert wird. Dies wäre z.B. bei Getreide, Milch, Rind- und Schweinefleisch der Fall.

\section{d) Betriebsvergrösserungen, Oekologieauflagen und Kostensen- kungen}

Von entscheidender Bedeutung ist der Zusammenhang $z$ wischen Betriebsgrösse und ökologischen Anforderungen an die Agrarproduktion und die Landschaft. Es ist bekannt, dass grössere Betriebe aus wirtschaftlichen Gründen extensiver als kleinere Betriebe produzieren. Die Betriebsleiter werden deshalb versuchen, Einkommenseinbussen - verursacht durch ökologische Auflagen und Preissenkungen - durch Betriebsvergrösserungen aufzufangen. Dadurch können die fixen betrieblichen Einrichtungen besser ausgelastet werden, was einer Senkung der Produktionskosten pro produzierter Einheit gleichkommt. Unter Berücksichtigung der Wirtschaftlichkeit werden also ökologisch geführte Betriebe dann überlebensfähig sein, wenn der Betriebsleiter nicht unter dem ständigen Druck zur Steigerung der Flächenintensität steht bzw. wenn die Betriebe sich flächenmässig vergrössern können. 
In nachfolgender Tabelle seien einige Kalkulationen aufgeführt, die grössenordnungsmässig bei GATT bzw. EG-Annäherungen nach und nach eintreffen dürften.

Tab. 1: Preis- und Kostenschätzungen bei GATT- und EG-Beitritten für durchschnittliche schweizerische Haupterwerbsbetriebe

\begin{tabular}{|c|c|c|}
\hline & $\begin{array}{l}\text { GATT-Uruguayrunde } \\
\text { bei Annahme der } \\
\text { heutigen EG-Offerte }\end{array}$ & $\begin{array}{l}\text { EG-Anschluss } \\
\text { Annahmen }\end{array}$ \\
\hline Produktpreissenkung & $(-) \quad 30 \%$ & $(-) 50$ \\
\hline $\begin{array}{l}\text { Kompensation durch } \\
\text { Kostensenkung wegen } \\
\text { Grösseneffekt (siehe } \\
\text { Abb.9) }\end{array}$ & (+) $15 \%$ & $(+) \quad 15 \%$ \\
\hline $\begin{array}{l}\text { Senkung der } \\
\text { Inputkosten (EG-Markt) }\end{array}$ & & $\begin{array}{l}\text { (+) } 20 \% \\
\text { (mindestens) }\end{array}$ \\
\hline $\begin{array}{l}\text { Kompensation über } \\
\text { verschiedene } \\
\text { Direktzahlungen }\end{array}$ & (+) $15 \%$ & (+) $15 \%$ \\
\hline
\end{tabular}

Es dürfte somit offensichtlich sein, dass vor allem tüchtige Bauern mit grösseren Betrieben gleichzeitig sowohl den ökologischen als auch den ökonomischen Erfordernissen der Zukunft gerecht werden können. Gute Ueberlebenschancen dürften langfristig im Durchschnitt jene Betriebe haben, die mindestens 25 bis 30 Hektaren rationell bewirtschaften können. Denn auf solche Betriebe dürfte sich die zukünftige Agrarpreispolitik - sowohl aus Gründen des Agraraussenhandels als auch der Oekologie - ausrichten. Unter diesen Annahmen gehen die Schätzungen dahin, dass die Zahl der Haupterwerbsbetriebe von heute rund 60'000 auf etwa 50'000 in 20 Jahren abnehmen wird. Kleineren Betrieben kann aus sozialen Gründen vorübergehend mit produktionsneutralen Direktzahlungen geholfen werden.

Neben diesem Blick auf die Entwicklung der Haupterwerbsbetriebe dürfen die Nebenerwerbsbetriebe nicht vergessen werden. Diese sind im Erscheinungsbild uneinheitlicher als Haupterwerbsbetriebe. Bei günstiger wirtschaftlicher Entwicklung ist der Sog zur Abwanderung grösser. Trotzdem wird eine grosse Zahl von Nebenerwerbsbetrieben bestehen bleiben. Voraussetzung dafür ist, dass der nicht-landwirtschaftliche Teil dieser Existenzen der wirtschaftlich tragende Teil ist. 
Abb. 9: Abnehmende Kosten bei zunehmender Kuhzahl

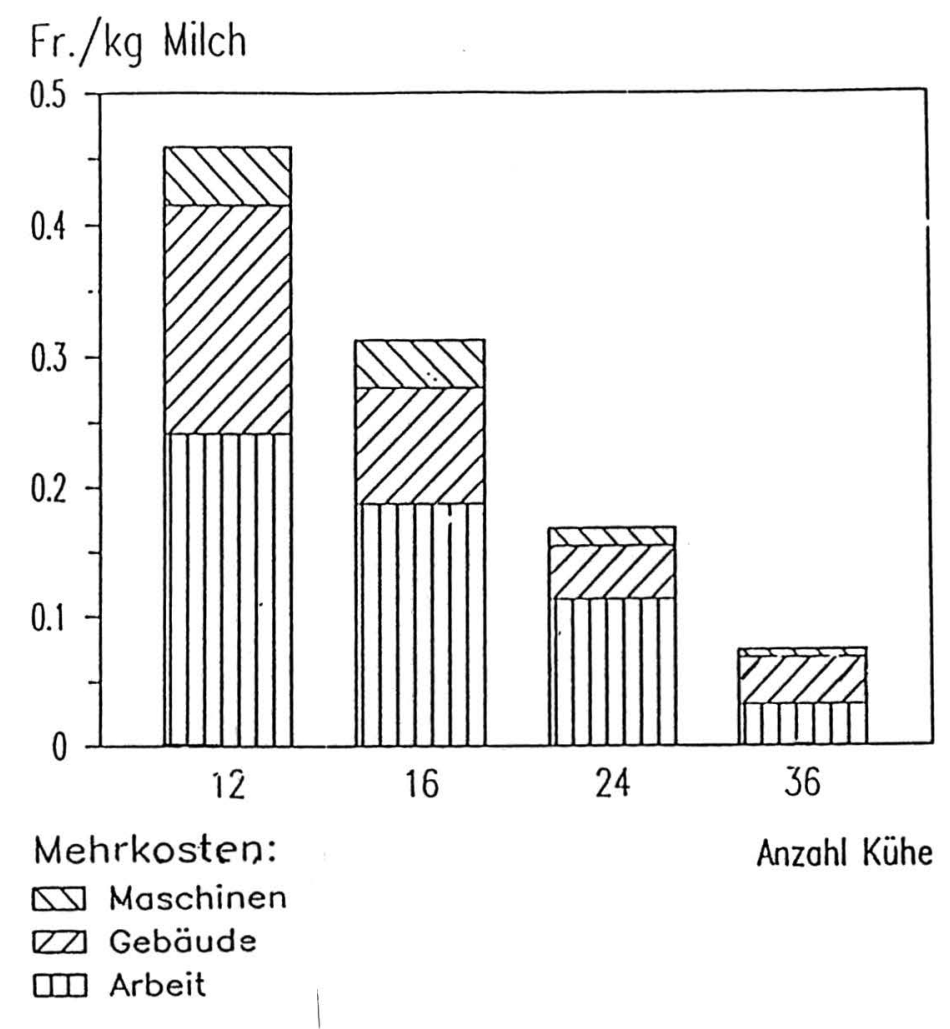

Quelle: Duttweiler et al., 1988

Die Entwicklungen der Betriebsgrössen zeigen uns die Abbildungen 10 und 11 . Vorerst sei auf Abbildung 10 hingewiesen, die nach dem Kriterium der Flächengrössenklassen die Hauptund Nebenerwersbetriebe aufzeigt. In Abbildung 11 werden nur die Grössenklassen der hauptberuflichen Betriebe fortgeschrieben. Dieser Darstellung liegt die Annahme zugrunde, dass die Abwanderung wie in den letzten 15 Jahren anhält und dass die Fläche sich proportional auf die jeweils grösseren Betriebsklassen verteilt. Die neusten Ergebnisse der Betriebszählung von 1990 bestätigen mit ca. 62'000 Haupterwerbsbetrieben die in Abbildung 11 aufgezeigte Entwicklung als relativ zuverlässig. Bei dieser Entwicklung bleiben die schweizerischen Betriebe im internationalen Vergleich noch klein und auf alle Fälle bleiben es Familienbetriebe. 
Abb. 10: Verteilung der Betriebe nach Grössenklassen

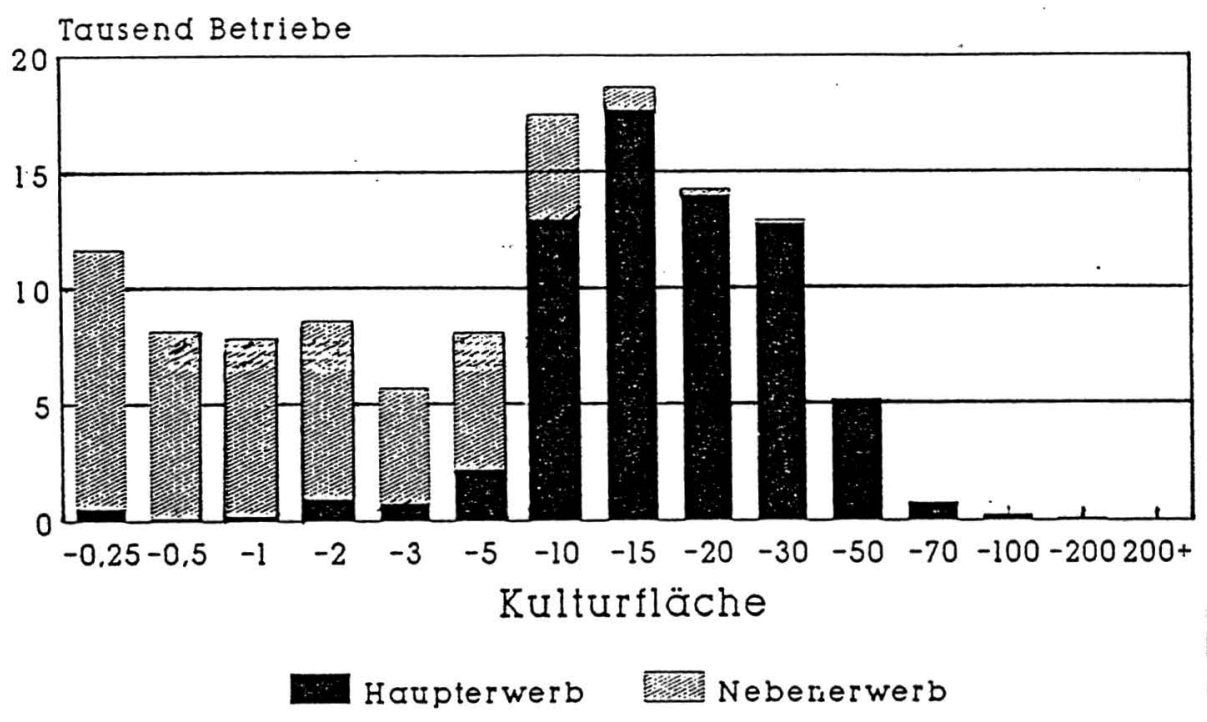

Quelle: Bundesamt für Statistik 1985, eigene Darstellung

Abb. 11: Verteilung der Haupterwerbsbetriebe nach Grössenklassen bei Fortschreibung vergangener Trends

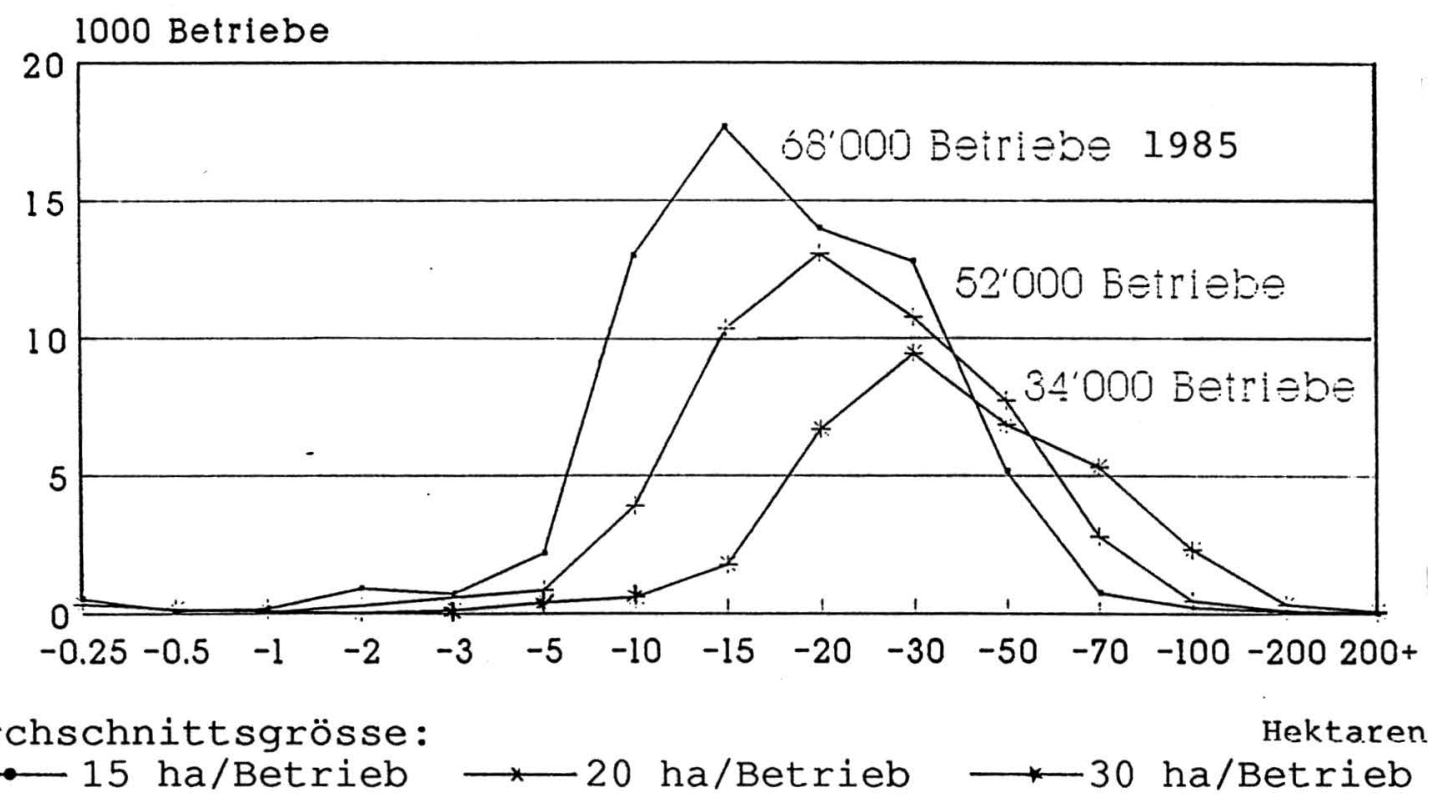

Quelle: Eigene Simulationsberechnungen

Hiezu soll mit zwei Abbildungen ein europäischer Vergleich der Grössenklassen angestellt werden. Abbildung 12 zeigt einen Vergleich der schweizerischen Grössenklassen mit drei EG-Ländern. Diese Abbildung enthält alle Betriebe. So ist insbesondere ersichtlich, dass die Schweiz proportional sehr viele kleine Nebenerwerbsbetriebe aufweist. Zweitens ist aber auch eindeutig zu sehen, dass in der Schweiz noch die Grössenklasse von 10-20 Hektaren dominiert, während dies bei den Vergleichsländern eindeutig Klassen von über 20 Hektaren 
sind. Diese Abbildung deutet somit in die Richtung, wohin sich wohl auch schweizerische Betriebe unter EG-Verhältnissen bewegen müssten.

Ganz besonders einleuchtend wird der Veränderungsdruck, wenn wir in Abbildung 13 die Haupterwerbsbetriebe der Schweiz und der BRD einander gegenüberstellen. Denn es werden die Haupterwerbsbetriebe sein, auf die die zukünftige Agrarpolitik ausgerichtet sein wird. Einschränkend kann beigefügt werden, dass die Betriebsgrösse nach Fläche sicher nicht der einzige Vergleichsmasstab ist, sicher aber einer der dominanten. Zudem schliesst die Darstellung die Spezialbetriebe aus.

\section{Abb. 12: Europäischer Grössenvergleich von Landwirtschafts- betrieben}

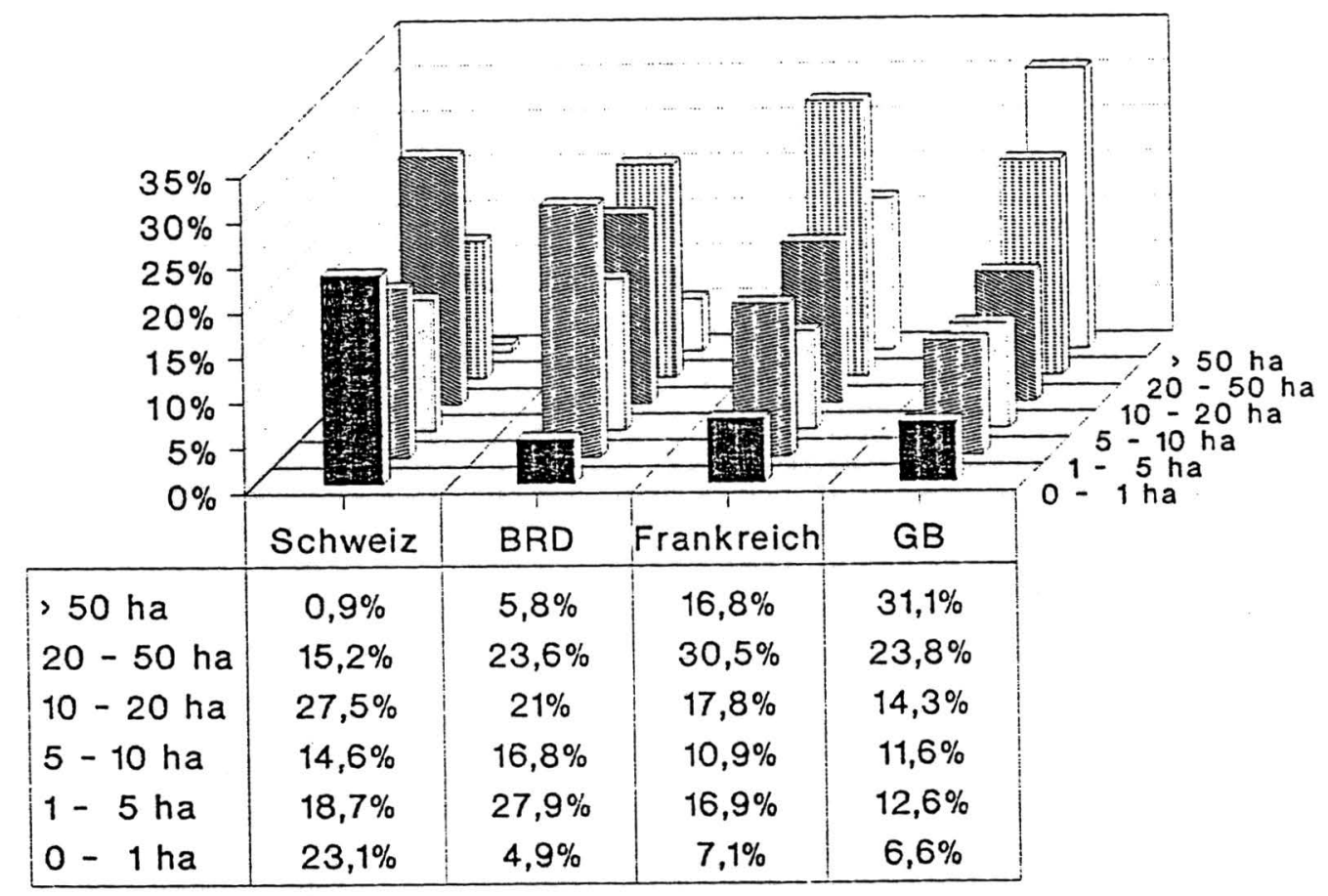

Quelle: Eidg. Betriebszählungen 1985, Statistisches Jahrbuch über Ernährung, Landwirtschaft und Forsten 1990 
Abb. 13: Haupterwerbsbetriebe nach Grössenklassen

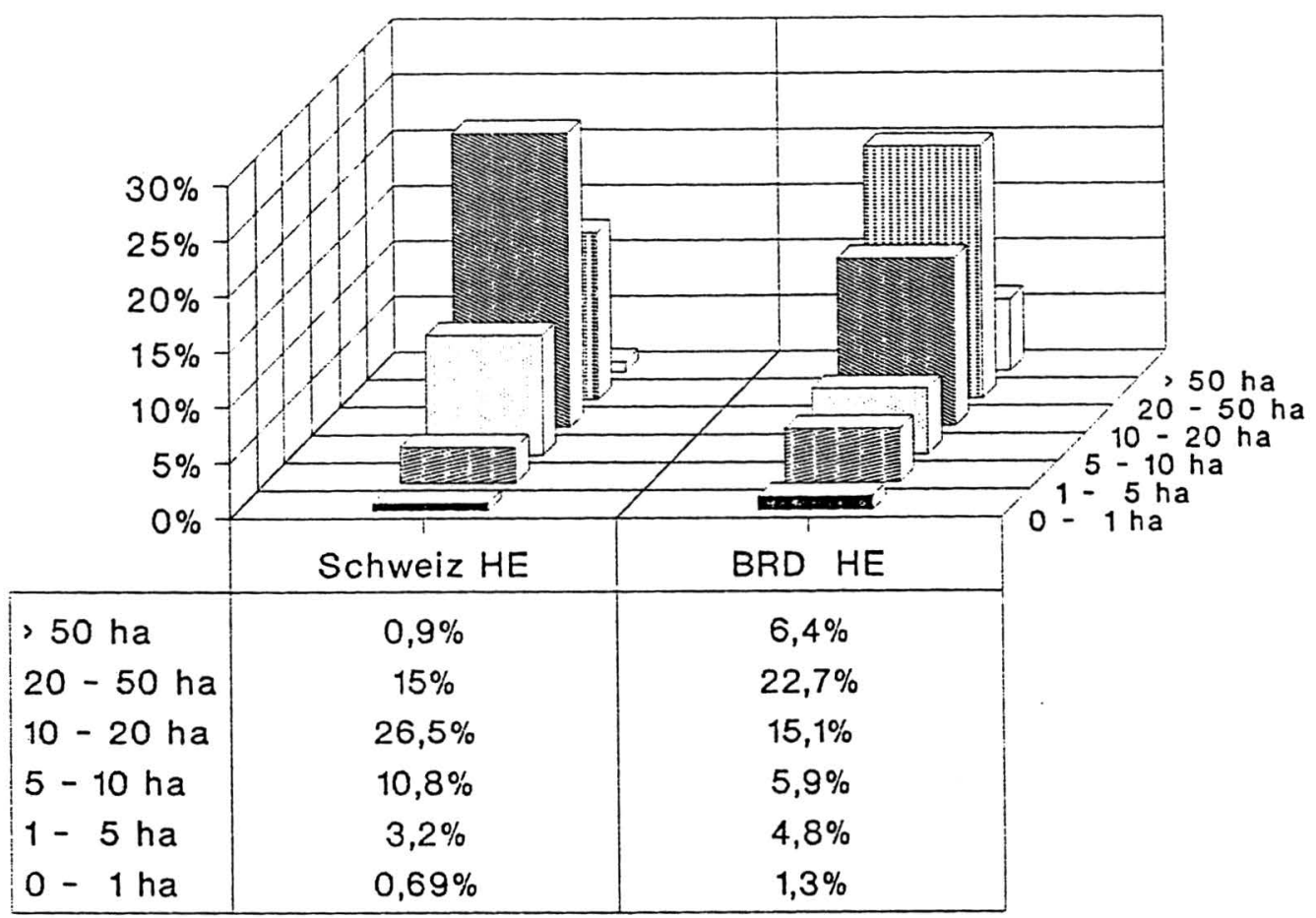

Ouelle: Eidg. Betriebszählungen 1985, Statistisches Jahrbuch über Ernährung, Landwirtschaft und Forsten 1990

\section{DAS NEUE LEITBILD: MULTIFUNKTIONALE IAANDWIRTSCHAFT, INSBESONDERE DES ALPENRAUMES}

Im Rahmen der GATT-Uruguay-Runde wird seitens der europäischen Bauernvertreter die Multifunktionalität der Landwirtschaft betont. Dieser Begriff besagt, dass dem Agrarsektor neben der Produktion von Nahrungsmitteln noch weitere Funktionen zufallen. Daraus wird die Schlussfolgerung gezogen, dass sich die Agrarpolitik des GATT nicht ausschliesslich an der Reorganisation (Liberalisierung) der Agrarmärkte und des Agrarproduktehandels orientieren dürfte, wie dies seitens der USA und der CAIRNS-Gruppe gefordert wird. Vielmehr müssten im Rahmen der zukünftigen GATT-Bestimmungen auch produktbezogene agrarpolitische Schutzmassnahmen erlaubt sein, welche darauf abzielen, diese zusätzlichen Aufgaben der Landwirtschaft zu ermöglichen.

Wir stellen diese Forderung ans GATT in Frage. Es ist, wie wir zeigen möchten, fraglich, ob über diesen Weg das erreicht würde, was die fordernden organisationen verlangen. Zudem ist der Begriff "Multifunktionlität" dermassen komplex bzw. es ist eine Leerformel, die ihren Inhalt erst durch konkrete Forderungen und Aktivitäten erhalten kann. Wir wählen daher einen streng analytischen zugang zu diesem neuen Begriff (mit altem Inhalt). Das Ziel dieser Analyse besteht darin, den Begriff "Multifunktionalität" für die praktische Politik brauchbar zu machen. 
Um eine zielgerichtete und effiziente multifunktionale Berggebietspolitik formulieren zu können, müssen wir erstens die verschiedenen Funktionen, welche die Berglandwirtschaft erfüllen soll, physisch kennen bzw. definieren. Zweitens müssen wir uns über die ökonomischen Eigenschaften dieser Funktionen im klaren sein. Drittens müssen die sozio-ökonomischen Ebenen, auf denen diese Funktionen verankert sind, identifiziert werden. Anhand der Abbildungen 14 und 15 wird auf diese drei Punkte eingegangen.

\section{Abb. 14: Die Funktionen der Landwirtschaft und ihre ökonomi- schen Eigenschaften}

\begin{tabular}{|c|c|c|c|c|}
\hline FUNKTIONEN & $\begin{array}{l}\text { Privates } \\
\text { Gut }\end{array}$ & $\begin{array}{l}\text { Oeffentl } \\
\text { Gut }(+I-)\end{array}$ & $\begin{array}{l}\text { Positive } \\
\text { Exter- } \\
\text { nalität }\end{array}$ & $\begin{array}{l}\text { Negative } \\
\text { Exter- } \\
\text { nalität }\end{array}$ \\
\hline Vorsorge Krisenzeiten & & $x$ & & \\
\hline $\begin{array}{l}\text { Preiswerte gesunde } \\
\text { Nahrungsmittel }\end{array}$ & $\mathrm{x}$ & & & \\
\hline Umweltgerechte Produktion & & $\mathrm{x}$ & $\mathrm{x}$ & \\
\hline Umweltbelastung & & $\mathrm{x}$ & & $\mathrm{x}$ \\
\hline Erhaltung Kulturlandschaft & & $\mathrm{x}$ & $\mathrm{x}$ & \\
\hline Dezentrale Besiedlung & & $\mathrm{x}$ & $\mathrm{x}$ & \\
\hline Erholungsraum & & $\mathrm{x}$ & $\mathrm{x}$ & \\
\hline Leistungsfähige Betriebe & $\mathrm{x}$ & & & \\
\hline Einkommenssicherung & $\mathrm{x}$ & & & \\
\hline Nationale Konkurrenzfähigkeit & $\mathrm{x}$ & & & \\
\hline $\begin{array}{l}\text { Internationale Konkurrenz- } \\
\text { fähigkeit }\end{array}$ & $\mathrm{x}$ & & & \\
\hline
\end{tabular}

Abbildung 14 zeigt eine Zusammenstellung der Funktionen, die der schweizerischen Landwirtschaft und insbesondere der Berglandwirtschaft zugeordnet werden können. Die Funktionen zeigen die vielfältigen Aufgaben und Anforderungen, die an die Landwirtschaft gestellt werden. Das Aufgabenspektrum reicht von der Vorsorge für Krisenzeiten über ökologische und ökonomische Anforderungen bis hin zur dezentralen Besiedlungsstruktur und internationalen Wettbewerbsfähigkeit. Die vielfältigen Funktionen der Landwirtschaft zeichnen sich nun durch unterschiedliche ökonomische Eigenschaften aus. Um die Diskussion weiter führen zu können, müssen wir zunächst die ökonomischen Eigenschaften definieren:

Private Güter sind Wirtschaftsgüter, die in einer Marktwirtschaft von privaten Anbietern direkt angeboten werden. Die effizienteste Verteilung erfolgt über den Markt. Diese Güter unterliegen dem Ausschlussprinzip. In diese Kategorie fallen beispielsweise landwirtschaftliche Produkte wie Getreide, Fleisch usw. 
Oeffentliche Güter sind Wirtschaftsgüter, deren Konsum nicht von der Zahlung eines Entgelts abhängig gemacht werden kann. Der den Konsumenten daraus zufliessende Nutzen ist unabhängig von der Zahl der Konsumenten. Es liegt klassisches Marktversagen vor. Die Entscheidung über die Schaffung eines öffentlichen Gutes ist deshalb stets das Ergebnis eines kollektiven Willensbildungsprozesses. Die effiziente Verteilung eines öffentlichen Gutes erfolgt auch nicht über den Markt (Marktversagen). Beispiele für öffentliche Güter sind Strassen, Parkanlagen, die Landesverteidigung, schöne Landschaften, usw.

Positive/negative Externalitäten sind Auswirkungen einer ökonomischen Tätigkeit auf Dritte, nicht am Produktionsprozess Beteiligte. Externalitäten entstehen durch Marktversagen, d.h. deren Nutzen oder Schaden schlägt sich nicht in Marktpreisen nieder. Manche, besonders die uns interessierenden Externalitäten, haben gleichzeitig den Charakter von öffentlichen Gütern oder Schäden. Eine positive Externalität ist beispielsweise auch die Pflege der Kulturlandschaft durch die Landwirte. Eine negative Externalität wäre konkret das Nitrat im Tinkwasser aufgrund $\mathrm{zu}$ intensiver landwirtschaftlicher Bodennutzung.

Aus diesen ersten zwei analytischen Schritten ziehen wir folgendes Fazit: Je nach ökonomischem Charakter der einzelnen Funktionen werden es jeweils unterschiedliche Massnahmen sein, die zu einer effizienten Ziel-/Funktionserfüllung führen (vgl. die Misserfolge in der Vergangenheit, z.B. bezüglich des hohen Milchpreises zur Erhaltung kleiner Bergbetriebe).

Der dritte analytische schritt besteht nun darin, dass die Funktionen jener sozioökonomischen Ebene zugewiesen werden, die davon direkt betroffen ist (Frey, 1981). Diese Ebenen sind nach Abbildung 15 der Landwirtschaftsbetrieb, das Dorf, die Region, der Kanton, die Schweiz, Europa bzw. die internationale Ebene. 
Abb. 15: Sozioökonomische Betroffenheitsebenen der landwirtschaftlichen Funktionen

\begin{tabular}{|c|c|c|c|c|c|c|}
\hline EUNKTION & Betrieb & Dorf & Region & Kanton & Schweiz & $\begin{array}{c}\text { Interna- } \\
\text { tional }\end{array}$ \\
\hline Vorsorge Krisenzeiten & & & & & $x$ & \\
\hline $\begin{array}{l}\text { Preiswerte gesunde } \\
\text { Nahrungsmittel }\end{array}$ & & & & & $\mathrm{x}$ & \\
\hline Umweltgerechte Produktion & & $x$ & $\mathrm{x}$ & & & \\
\hline Umweltbelastung & & $\mathrm{x}$ & $x$ & & & \\
\hline Erhaltung Kulturlandschaft & & $\mathrm{x}$ & & & & \\
\hline $\begin{array}{l}\text { Dezentrale Konkurrenz- } \\
\text { fähigkeit }\end{array}$ & & $\mathrm{x}$ & $\mathrm{x}$ & & & \\
\hline Erholungswert der LW & & $\mathrm{x}$ & & & & \\
\hline Leistungsfähige Betriebe & $\mathrm{x}$ & & & & & \\
\hline Einkommenssicherung & $\mathrm{x}$ & & & & & \\
\hline $\begin{array}{l}\text { Nationale Konkurrenz- } \\
\text { fähigkeit }\end{array}$ & & & $\mathrm{x}$ & $x$ & & \\
\hline $\begin{array}{l}\text { Internationale Kon- } \\
\text { kurrenzfähigkeit }\end{array}$ & & & & & $\mathrm{x}$ & $\mathrm{x}$ \\
\hline
\end{tabular}

Die Kolonnen in Abbildung 15 enthalten angekreuzte Felder, in denen die Funktionen beheimatet sind. So ist der Einzellandwirt der Betroffene bezüglich Leistungsfähigkeit und Einkommen. Umweltgerechte Produktion, Umweltbelastung, Biologische Vielfalt und Erholungsraum sind (in unserem Zusammenhang) dorfbezogen oder fallweise regionalbezogene Funktionen. Krisenvorsorge, preiswerte und gesunde Nahrungsmittel sind nationale Funktionen, und die nationale und internationale Konkurrenzfähigkeit ist die Brückenfunktion zur internationalen Ebene.

Nun bleibt die Frage im Raum, wie die Einzelaspekte sich in ein handlungsorientiertes Konzept umsetzen lassen. Wir halten vorerst nochmals die schritte fest:

1. Die Funktionen sind physisch zu definieren bzw. zu umschreiben.

2. Der ökonomische Charakter der Funktionen ist aufzuzeigen.

3. Die Funktionen sind jeweils den Ebenen zuzuordnen, wo die Betroffenheit der Bürger am grössten ist.

Damit ist die Multifunktionalität umschrieben und charakterisiert. Es folgt nun die Stufe der Massnahmen zur Erfüllung der Funktionen. Als Kriterien postulieren wir Verhältnismässigkeit und Effizienz. Ferner halten wir fest, dass alle Funktionen grundsätzlich wichtig sind. Sowohl die individuellen wie die öffentlichen Ziele/Funktionen sind zu beachten. 
Zweites Fazit, aufbauend auf dem ersten lautet somit: Massnahmen haben - dem ökonomischen Charakter der Funktion entsprechend - auf die einzelnen Ebenen ausgerichtet zu sein, z.B.: Preisbeschlüsse bezüglich internationaler Konkurrenzfähigkeit; z.B. Flächenbeiträge bezüglich Flächenbewirtschaftung in Regionen; z.B. Meliorationen bezüglich Agrarstrukturen in Dörfern; z.B. Einkommenszahlungen und Investitionskredite bezüglich Einzelbetriebsstrukturen.

Negativ formuliert kann anderseits gesagt werden, dass z.B. hohe Produktpreise negativ bezüglich der Intensität der landwirtschaftlichen Produktion (zuviel Tiere und Dünger pro Hektare) wirken. Ebenso schaden sie auch der internationalen Konkurrenzfähigkeit und führen zu starken Verzerrungen der Agrarmarktlage zuungunsten der Entwicklungsländer und der kleinen Landwirtschaftsbetriebe (indem die grösseren davon immer überproportional begünstigt werden). Oder: GATTEntscheidungen können nicht auf Umweltfunktionen tieferer Ebenen ausgerichtet werden. Diese sind mit effizienten Massnahmen Ebene-gerecht zu erfüllen. GATT-Verträge ihrerseits haben also den internationalen warenaustausch zu ermöglichen.

Die Erhaltung der alpinen Kulturlandschaft ist somit $\mathrm{zu}$ erreichen, indem nach Ebenen gerechte Politik betrieben wird, wie etwa der bei der Raumplanung beschrittene Weg. Dieser Weg lässt zeit- und problembedingte Differenzierungen zu und kann entsprechend ausgestaltet werden. Aus den Fehlern der Vergangenheit ist zu lernen - für das Schweizer Berggebiet waren die Fehler zum Glück dank vielfältigen Direktzahlungen und Strukturhilfen (Regional- und Agrarpolitik) nicht allzu gross.

FOLGERUNGEN/ZUSAMMENFASSUNG ZUR ERHALTUNG DER BÄUERIICHEN KULTURLANDSCHAFT DER ALPEN

1. These: Internationale Verhandlungen (EG, EWR, GATT) führen zu Verunsicherungen. Neue Begriffe prägen die Diskussion, so z.B. Multifunktionalität der Landwirtschaft: Deren Inhalt ist vielfältig.

2. These: Im historischen Zeitablauf betrachtet hat sich die Kulturlandschaft im Alpenraum stark verändert: Rodung im Mittelalter, Abholzung im 19. Jahrhundert (mit Flutkatastrophe 1868 und Lawinen). Folge: erstes Forstgesetz mit Artikel über Walderhaltung. Zur Zeit Polarisierung der Nutzung wegen Bergmechanisierung bei mangelnder Erschliessung.

3. These: Minimale Dorfgrössen mit sozio-ökonomischem Gleichgewicht bilden Voraussetzung für Bauernbetriebe (Dorfmodell). 
4. These: Der "Fehler" von Art. 29 LwG (kostendeckende Preise) ist durch Spezialgesetze mit zunehmend produktionsungebundenem Charakter sowie mit Strukturverbesserungsmassnahmen korrigiert worden.

5. These: Zielvorstellungen der Einzelbauern (befriedigende Einkommen, normale Arbeitsbelastung, soziale Integration, Sinnerfüllung in der Arbeit) sind notwendige Voraussetzungen zur Erreichung der Funktionen mit öffentlichem Charakter.

6. These: Die vorgesehene Erweiterung des Landwirtschaftsgesetzes mit Art. $31 \mathrm{a}$ und $31 \mathrm{~b}$ geht mit der Kompetenz für einkommens- und umweltbezogene Massnahmen in die richtige Richtung. Strukturverbesserungen unterstützen deren Wirkungsweise.

7. These: GATT- und EG-Annäherungen führen mittelfristig zu einem Abbau des produktionsbezogenen Agrarschutzes. Für den Alpenraum können gleichzeitig funktionsbezogene Direktzahlungen ausgebaut werden. Korrekturen im heutigen system sind möglich (Ueberschüsse, zu hohe Intensität).

8. These: Multifunktionalität der Landwirtschaft enthält als Begriff einen alten Inhalt, ist aber brauchbar, sofern konsequent nach den geforderten vielen Funktionen eine effiziente Politik betrieben wird.

Die Funktionen unterscheiden sich nach

- physischen Eigenschaften

- ökonomischen Eigenschaften

- Ort der Relevanz, betroffene Ebene

Eine zukünftige Politik, nach stärkerer Gewichtung dieser Aspekte, kann die Kulturlandschaft im Alpenraum grundsätzlich fördern.

9. These: Kulturlandschaft bzw. Multifunktionalität der Landwirtschaft im Alpenraum bedarf eines differenzierten funktions- und strukturspezifischen Massnahmenpaketes, das effizient die Kongruenz zwischen den Zielsystemen des Einzelbauern und der Oeffentlichkeit erreicht. 


\section{LITERATURVERZEICHNIS}

Abel, W., Agrarpolitik, Göttingen-Verlag Vandenhoeck und Ruprecht, Band 11, S. 20, 1951

Anwander, S., Bürgi, St., Cavegn, G., Meyer, L., Rieder, P., Salmini, J., Direktzahlungen an die Landwirtschaft, vdf. Zürich, 1990

Bernegger, U., Die Strukturentwicklung der Berglandwirtschaft am Beispiel des Kanton Graubünden - Ein Modell zur quantitativen Wirkungsanalyse agrarwirtschaftlicher Massnahmen, V Diss. ETH Nr. 7740, Zürich, 1985

Bernegger, U., Cavegn, G., Der Beitrag der Landwirtschaft zur Erhaltung von Bauerndörfern im Berggebiet, in: Landwirtschaft Schweiz, Band 2, (8), 1989

Bernegger, U., Cavegn, G., Meyer, L., Rieder, P., Existenzfähige Bergbauernbetriebe als Voraussetzung für die Nutzung von Grenzertragsböden und einer gesicherten Besiedlung in-nicht-touristischen Bergdörfern, Studie im Rahmen des NFP 22 "Boden", Institut für Agrarwirtschaft ETH, Zürich, 1990

Bundi, M., Zur Besiedlungs- und Wirtschaftsgeschichte Graubündens im Mittelalter, Calvenverlag, 1982

Duttweiler, R., Ammann, H., Hilty, R., Naef, E., Der Einfluss der Kuhherdengrösse auf die Kosten der Milchproduktion, Landwirtschaft Schweiz, Band 1 (4), 1988

Frey, B., Theorie der Wirtschaftspolitik, Vahlen-Verlag, München, 1981

Howald, O., Einführung in die Agrarpolitik, Verlag A. Frauke AG, Bern, S. 31, 1946

Löhr, L., Bergbauernwirtschaft im Alpenraum, LeopoldStocker-Verlag, Graz, Stuttgart, S. 14, 1971

Rieder, P., Egger, U., Flückiger, St., Schweizerische Agrarmärkte, vdf. Zürich, 1992

Rieder, P., Direktzahlungen an die Landwirtschaft - Ein agrar-ökonomisches Konzept, Landwirtschaft Schweiz Vol. $1,(7)$, S. 401-404, 1988

Rieder, P., Landwirtschaft und Besiedlung in Graubünden, in: Beitäge zur Geographie Graubündens, NPOC, 1991

von Siebenthal, w., Direktzahlungen als Mittel der landwirtschaftlichen Einkommenspolitik, Diss., Universität Bern, 1986

Sprecher, v., Jenni, R., Kulturgeschichte der Drei Bünde, Verlag Bischofsberger, Chur, 1976

Zinsli, P., Walser Wachstum, Verlag Huber, Frauenfeld, 1968 
Institut für Agrarwirtschaft

\section{Schriftenreihe/Publications}

Jahr/ Titel und Autor/

année Titre et auteur

1991/1 Prioritäten und Transparenz in der landwirtschaftlichen Forschung Peter Rieder
Seiten/ Preis/

pages prix

19 5.-

32 10.-

$16 \quad 5 .-$

$14 \quad 5 .-$

32 10.-

65

10.rural en Europe

Erwin Stucki

1992/1 Agrarstrukturentwicklungen in der Schweiz unter

14 5.-

Aspekten nationaler Agrarpolitik und laufender internationaler Verhandlungen

Peter Rieder

1992/2 Le développement dans la Vallée des Ormonts: quel rôle pour l'agriculture de montagne dans le développement local?

Erwin Stucki

$1992 / 3$

IER-CERME / Rapport d'activité 1991 / Programme 1992 Erwin Stucki

16 5.-

30 10.-

1992/4 Sustainable Development in Rural Areas

Some Methodological Issus

55 10.- 


\section{BESTELLUNG/COMMANDE}

Name und Vorname/

Nom et prénom :

Adresse :

Schrift Nr./publication no :

Einsenden an/ envoyer à :

\author{
IAW/ETHZ, Sekretariat \\ ETH-Zentrum, 8092 ZURICH
}

